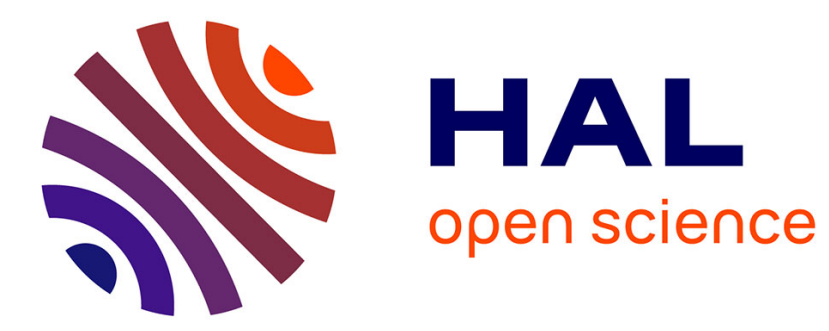

\title{
Long-term endogenous economic growth and energy transitions.
}

\author{
Victor Court, Pierre-André P.-A. Jouvet, Frédéric Lantz
}

\section{To cite this version:}

Victor Court, Pierre-André P.-A. Jouvet, Frédéric Lantz. Long-term endogenous economic growth and energy transitions.. Energy Journal, 2018, 39 (1), 10.5547/01956574.39.1.vcou . hal-01987974

\section{HAL Id: hal-01987974 \\ https://hal-ifp.archives-ouvertes.fr/hal-01987974}

Submitted on 12 Feb 2019

HAL is a multi-disciplinary open access archive for the deposit and dissemination of scientific research documents, whether they are published or not. The documents may come from teaching and research institutions in France or abroad, or from public or private research centers.
L'archive ouverte pluridisciplinaire HAL, est destinée au dépôt et à la diffusion de documents scientifiques de niveau recherche, publiés ou non, émanant des établissements d'enseignement et de recherche français ou étrangers, des laboratoires publics ou privés. 
WWW.IAEE.ORG

The following article is a preprint of a scientific paper that has completed the peer-review process and been accepted for publication within The Energy Journal.

While the International Association for Energy Economics (IAEE) makes every effort to ensure the veracity of the material and the accuracy of the data therein, IAEE is not responsible for the citing of this content until the article is actually printed in a final version of The Energy Journal. For example, preprinted articles are often moved from issue to issue affecting page numbers, and actual volume and issue numbers. Care should be given when citing Energy Journal preprint articles. 


\title{
Long-Term Endogenous Economic Growth and Energy Transitions
}

\author{
Victor Court, ${ }^{\mathrm{abc} *}$ Pierre-André Jouvet, ${ }^{\mathrm{ab}}$ and Frédéric Lantz ${ }^{\mathrm{c}}$
}

\begin{abstract}
This article builds a bridge between the endogenous economic growth theory, the biophysical economics perspective, and the past and future transitions between renewable and nonrenewable energy forms that economies have had to and will have to accomplish. We provide an endogenous economic growth model subject to the physical limits of the real world, meaning that nonrenewable and renewable energy production costs have functional forms that respect physical constraints, and that technological level is precisely defined as the efficiency of primary-touseful exergy conversion. The model supports the evidence that historical productions of renewable and nonrenewable energy have greatly influenced past economic growth. Indeed, from an initial almost-renewable-only supply regime we reproduce the increasing reliance on nonrenewable energy that has allowed the global economy to leave the state of economic stagnation that had characterized the largest part of its history. We then study the inevitable transition towards complete renewable energy that human will have to deal with in a not-too-far future since nonrenewable energy comes by definition from a finite stock. Through simulation we study in which circumstances this transition could have negative impacts on economic growth (peak followed by degrowth phase). We show that the implementation of a carbon price can partially smooth such unfortunate dynamics, depending on the ways of use of the income generated by the carbon pricing.
\end{abstract}

Keywords: Endogenous economic growth, Technological change, Exergy, Energy transition.

https://doi.org/10.5547/01956574.39.1.vcou

\section{INTRODUCTION}

Compared to previous millennia, human societies have experienced tremendous increases of development over the past two hundred years (Maddison, 2007). This period has been indeed mostly marked by increasing technological and material production improvements, which have however spread unequally through the different parts of the world. This pattern is commonly measured by the growth rate of the gross world product (GWP) at global scale, or gross domestic product (GDP) at national level. Despite many critics regarding the imperfection of these indicators, they continue nowadays to attract most of the attention of economists, policy makers, and media.

a EconomiX, UMR 7235, UPL, Univ. Paris Nanterre, 200 avenue de la République, 92001 Nanterre cedex, France.

b Chaire Economie du Climat, Palais Brongniart, 28 Place de la Bourse, 75002 Paris, France.

c IFP Energies Nouvelles, 1-4 avenue de Bois Préau, 92852 Rueil-Malmaison cedex, France.

* Corresponding author. E-mail: victorcourt@free.fr.

The Energy Journal, Vol. 39, No. 1. Copyright (C) 2017 by the IAEE. All rights reserved. 


\subsection{Economic Growth Theories}

Since the development of the Solow-Swan model of exogenous economic growth (Solow, 1956; Swan, 1956), an important literature has been developed to propose different mechanisms for the endogenous origin of technological change: physical capital spillovers (Romer 1986), human capital spillovers (Lucas, 1988), research and development fueled by scarce physical capital (Romer 1987) or potentially infinite human capital (Romer, 1990), Schumpeterian creative destruction (Grossman and Helpman, 1991; Rebelo, 1991; Aghion and Howitt, 1992). These conceptual models must be understood as short-term explanations for contemporary growth and are not designed to explain how humanity was able to leave the state of economic stagnation that has characterized the largest part of its history to reach the contemporary regime of high technological and economic growth. This point has led to a search for a Unified Growth Theory (UGT) as coined by Galor (2011). The purpose of this unified theory of economic growth is to capture in a single formulation the Malthusian Epoch of population and economic growth stagnation, the take-off of these two variables in a Post-Malthusian Regime, and the Modern Growth Regime of sustained per capita income growth and decreasing population growth. According to Galor and Weil (2000) the principal mechanism explaining the transition between these three states is that above a certain threshold of population size the rate of technological change is sufficiently high to induce an increase in the importance of education to cope with the rapidly changing technological environment, which trigger the substitution of quality for quantity in child rearing and launch the economy on a virtuous cycle of human capital accumulation, technology improvement, and economic production increases. ${ }^{1}$ Despite the tremendous new insights brought by the current UGT, it is clear that similarly to the different models previously cited, it suffers (so far) from the same drawbacks, namely that (i) technological change is rather imprecisely defined in these models and most of the time stands as a time-dependent multiplier (or Total Factor Productivity ${ }^{2}$ ) of the aggregated macroeconomic production function; and more generally (ii) these theories do not take into account the fact that the economic system must necessarily follow the natural laws of the broader biophysical system in which it is embedded, and in particular the laws of thermodynamics.

\subsection{Goal and Content}

It is the purpose of the present article to propose a theoretical model of long-term endogenous economic growth that takes into account the underlying physical essence of the economic system. In section 2 we precise the background literature and the theoretical positioning of our work. We present in section 3 the model of a decentralized economy in which the accessibility of primary nonrenewable and renewable energy (in fact exergy), and the efficiency with which those inputs are converted into useful energy (exergy) services, determine the production of a final output good that is consumed or saved to allow investment. In section 4 we precise the calibration procedure to global historical data and show that the model adequately reproduce, from 1750 to 2010 , the pattern of historical global energy productions, technological change, and economic growth.

1. The canonical UGT model of Galor and Weil (2000) has been enhanced in several ways, see Galor (2011).

2. Such abstract representation of technological change aggregate very different production-augmenting factors such as: the primary-to-final and final-to-useful energy conversion efficiency (if energy is considered as an input factor), the division and organization of labor, the broader organization and efficiency of markets, the skill improvements of laborers, the contribution of information and communication technologies, but also the beneficial effects of inclusive institutions (which, for example, protect private property rights and consequently incentivize innovation and R\&D). 
We then run simulations of the model in order to study its dynamics in future times, in particular we assess the necessary conditions for a smooth transition towards an almost-renewable-only regime. We analyze in section 5 the interest of the implementation of a price on the polluting emissions of nonrenewable energy in order to smooth the transition towards increasing renewable energy in an original simulation setting in which the energy transition has negative impacts on economic growth. We conclude our work and discuss some of our hypotheses for further research developments in section 6 .

\section{LITERATURE REVIEW}

\subsection{Biophysical Economics}

The possibility that the finiteness of Earth's resources may limit future economic growth was formally addressed by Meadows et al. (1972). First responses to this study concerned methodological aspects, which unfortunately rapidly led to a rather unproductive debate. Nevertheless, this controversy led neoclassical economists to include a natural resource input in their theoretical models. For economic growth to continue forever in a finite physical world, neoclassical authors had to postulate in their models that human-made capital would ultimately be a perfect substitute for the natural resource input, or that technological change would have to be infinite in the future (Solow, 1974; Stiglitz, 1974; Dasgupta and Heal, 1974), or that macroeconomic value added would have to become increasingly dematerialized and based on knowledge (Smulders, 1995). These responses were clearly not satisfying for biophysical economists who viewed human society as a thermodynamic system (Georgescu-Roegen, 1971, 1979; Odum 1971, 1973; Daly, 1977; Cleveland et al., 1984), and for whom the capacity of the economy to increase its entropy production towards the broader environment is the main mechanism explaining apparent economic growth (Kümmel, 1989; Ayres, 1998). In other words, biophysical economists state that economic growth is primarily determined by the ability of societies to collect high-quality primary energy (defined by a high exergy content), convert it in useful energy (in fact exergy in the form of light, heat, motion and electricity), and in doing so reject low quality energy (i.e. low exergy/high entropy) in their broader environment (Ayres and Warr, 2009; Kümmel, 2011). Indeed, as repeatedly stressed by some authors (Ayres and Warr, 2009; Warr and Ayres, 2012), what is commonly called energy in economic studies and models is in fact exergy. Exergy is the valuable part or, more formally the potentially useful part of energy that can generates actual work, ${ }^{3}$ it is therefore a measure of the quality of energy. As required by the first law of thermodynamics, energy is conserved in the economic process. On the other hand, the second law of thermodynamics stipulates that exergy is degraded through the functioning of the economic system since it is composed of multiples irreversible processes that imply some entropy creation. Energy enters the economy as a high quality (high exergy content) input in the forms of fossils fuels, nuclear energy, and concentrated solar energy (biomass and water/wind flows). Those energy forms are ultimately dissipated into a lower-quality (lower exergy content) heat output that potentially contains zero exergy (and thus zero ability to generate useful work) if its temperature is the same as the broader environment. Hence, it is the exergy content of energy that constitutes a production factor used up in the economic process and not energy per se.

3. More precisely it is the maximum work that can be done by a system reversibly approaching thermodynamic equilibrium (Ayres and Warr 2009). 
In the remainder of this article we will sometimes stick to the familiar term of energy, even if, strictly speaking, we refer to exergy.

From a more practical point of view, biophysical economists have focused their attention on the different energy-return-on-investment (EROI) of energy systems. The EROI is the ratio of the quantity of energy delivered by a given process to the quantity of energy consumed (i.e. energy invested) in this same process. Hence, the EROI is a measure of the accessibility of a resource, meaning that the higher the EROI, the greater the amount of net energy (produced minus invested energy) delivered to society (Hall et al., 2014). For biophysical economists it makes no doubt that the development of industrial economies has been largely dependent on fossil fuels and in particular on their high EROI and consequent capacity to deliver large amounts of net energy to society. Despite an important literature, people working on net energy and EROI concepts have never developed aggregated models able to assess the impact of the changing energy supply (i.e. energy transitions) on the societal EROI and the economic growth dynamics. An exception to this fact is the GEMBA model of Dale et al. (2012) that incorporates a dynamic EROI function into an aggregated simulation model but without any specification as to agents' behaviour, and thus it completely differs from neoclassical optimal growth models.

\subsection{Energy Transition in the Neoclassical Framework}

On the other hand, several studies have focused on the transition between a nonrenewable and a renewable natural resource in a neoclassical analytical framework, but none of them refers to biophysical concepts such as exergy and EROI. Some of these studies (Jouvet and Schumacher, 2011; Hartley et al., 2016) are not able to have a simultaneous use of nonrenewable and renewable energy but only successive regimes that use specifically one of these energy forms, which logically generates some energy crisis behavior at the time of the abrupt switch. Conversely, the optimal growth model of Tahvonen and Salo (2001) is able to represent for an abstract economy a first phase of economic development that only rely on renewable energy, a second phase where renewable and nonrenewable energy are simultaneously used, and a third phase where the share of nonrenewable energy decreases because of increasing extraction costs, thus leading to a society that relies on renewable energy only. In Tsur and Zemel (2005) the attention is more focused on the $\mathrm{R} \& \mathrm{D}$ investments that allow a reduction in the cost of use of a backstop technology, but the broader effect of an energy transition on economic growth is not studied. Acemoglu et al. (2012) have studied the importance of the substitutability between nonrenewable and renewable inputs in directing endogenous technical change, and the influence of the optimal mix of environmental policies between carbon tax and R\&D subsidy.

\subsection{Bridging a Theoretical Gap}

In light of what has been presented so far it is clear that the purpose of the present article is to build a bridge between the different literatures related to: the endogenous economic growth theory, ${ }^{4}$ the biophysical economics perspective, and the transition between nonrenewable and re-

4. It is worth emphasizing that we do not consider our model to be an acceptable Unified Growth Theory model because we do not represent the population dynamics and its relation to human capital formation. One should consider the present paper as an effort to properly include energy in a very long-term endogenous economic growth model with the hope of producing a satisfactory energy-based unified theory of economic growth in future researches. 
newable energy forms. In fact, Fagnart and Germain (2014) have started to bridge this gap (though without referring to exergy) in a working paper in which the possibility of a smooth transition from nonrenewable to renewable energy and its impact on the EROI and economic growth is studied. In this model, uncalibrated simulations can only be done with an initial economy just before the nonrenewable energy peak and no production of renewable energy (which is thus not representative of reality). Hence, despite its novelty, there is different features of this model that we would like to address in the present paper, namely that (i) the nonrenewable energy is extracted without any capital requirement and consequently presents an infinite EROI, (ii) the backstop technology has a constant capital requirement per unit of energy output, (iii) technological change is bounded but completely exogenous, and (iv) the production function in the final good sector is of Leontief type. In order to address these particular settings and others mentioned earlier, we provide an endogenous economic growth model subject to the physical limits of the real world, meaning that nonrenewable and renewable energy production costs have functional forms that respect physical constraints, and that technological change is precisely defined as gains in the efficiency of primary-to-useful exergy conversion. Our model is consequently able to produce an increasing reliance on nonrenewable energy from an initial almost-renewable-only regime, and the subsequent inevitable transition towards renewable energy that human will have to deal with in a not-too-far future since nonrenewable energy comes by definition from a finite stock.

\section{STRUCTURE OF THE MODEL}

\subsection{Economic Product Allocation and Profit Maximization of Producers}

\section{Economic production allocation between sectors}

At each period $t$, the representative household receives the entire macroeconomic income made of the rents from total capital $K_{t}$ loaned at price $v_{t}$ and the different profits $\Pi_{t}, \Omega_{t}, \Psi_{t}$ of the respective nonrenewable energy (NRE), renewable energy (RE), and final good sectors. This total income is logically equal to the macroeconomic product $Y_{t}$, so

$$
Y_{t}=v_{t} K_{t}+\Pi_{t}+\Omega_{t}+\Psi_{t}, \quad \forall t \in\{0, \ldots, T\}
$$

The capital stock of the economy $K_{t}$ should not be considered as pure physical capital but rather as labor activated effective capital services since we do not represent the population and labor dynamics. Labor activated means that the capital services should be understood as the output result of the aggregation (in a production function that we do not explicit) of pure physical capital with routine labor hours provided by the population. Effective means that the capital services output also contain some human capital in the form of skills and hand-eye coordination (to which the recent contribution of information and communication technologies should be added). Given that we wish to calibrate the model on global historical data for the period 1750-2010, and then pursue simulation up to the point where nonrenewable energy is almost not used, we assume for simplicity a unitary depreciation rate of capital, implying that the time period $t_{\text {length }}$ between $t$ and $t+1$ corresponds to the average capital lifetime set to 20 years. As a consequence, it is acceptable to not represent any maximization behavior of the intertemporal welfare of the households, and rather to consider that the representative household consumes from the macroeconomic output $Y_{t}$ what is left over after the investment $I_{t}$ has been fulfilled. Hence, with $C_{t}$ representing the discretionary consumption at the macroeconomic level, we have 


$$
Y_{t}=\mathrm{C}_{t}+\mathrm{I}_{t}, \quad \forall t \in\{0, \ldots, T\}
$$

This means that the cost of capital services is in fact constant ${ }^{5}$ and worth $\nu \equiv(1+\mu)^{t_{\text {length }}} / \lambda$, where $\mu$ corresponds to the annual real interest rate of the economy, and $\lambda>0$ represents the productivity of the transformation of investments goods into productive capital. The dynamics of the capital investment level is

$$
I_{t}=\frac{K_{t+1}}{\lambda}
$$

Furthermore, equilibrium on the capital market must hold at each time period. Hence, the total capital stock of the economy $K_{t}$ is the sum of the NRE sector capital $Z_{t}$, the RE sector capital $G_{t}$, and the final good sector $H_{t}$.

$$
K_{t}=\left\{\begin{array}{c}
Z_{t}+G_{t}+H_{t}, \quad \forall t \in\left\{0, \ldots, T_{e}\right\} \\
G_{t}+H_{t}, \quad \forall t \in\left\{T_{e}+1, \ldots, T\right\} .
\end{array}\right.
$$

Where $T_{e}$ is the final time period of nonrenewable energy resource exploitation.

\section{Profit maximization of NRE producer}

The ultimately recoverable resource (URR) $\mathcal{R}$ represents the total amount of accessible primary nonrenewable energy in the Earth underground and exploited up to period $T_{e}$ by a representative price-taking firm. It is assumed that the representative firm does not know $\mathcal{R}$ but observe that its production cost evolves as the nonrenewable resource is progressively depleted. Extracting the annual gross primary nonrenewable energy quantity $R_{t}$ implies to consume some capital services $Z_{t}$. Furthermore, a fraction $0<\chi_{N R E}<1$ of the gross primary production $R_{t}$ is self-consumed by the NRE sector. Accordingly, in each period $t$ the NRE producer chooses an amount of capital services $Z_{t}$ in order to supply the quantity $R_{t}\left(1-\chi_{N R E}\right)$ of available primary nonrenewable energy to the final good sector at the unitary price $p_{t}$. Hence, the producer solves

$$
\max _{R_{t}, Z_{t}} \Pi_{t}=\left(1-\chi_{N R E}\right) p_{t} R_{t}-v Z_{t}, \quad \forall t \in\left\{0, \ldots, T_{e}\right\}
$$

under constraint,

$$
Z_{t}=\left(R_{t} D_{t}\right)^{\frac{1}{\theta}}, \text { with } 0<\theta<1 \quad \forall t \in\left\{0, \ldots, T_{e}\right\} .
$$

Where $D_{t}$ represents the capital intensiveness of the extraction process (i.e. the capital requirement per unit of gross primary NRE output), whose detailed definition is given in section 3.3. $0<\theta<1$ means that returns to scale are decreasing in the NRE sector. Recalling that $t_{\text {length }}$ is the time period length in years between $t$ and $t+1$, we have

5. Introducing the intertemporal welfare optimization behavior of the representative household implies a non-constant capital cost. This fact greatly complicates the calibration procedure but given the time frame chosen for the simulation it only smoothes the results without changing any of the qualitative outcomes of the model. 


$$
\sum_{t=0}^{T_{e}} t_{\text {length }} R_{t} \leq \mathcal{R}, \quad \forall t \in\left\{0, \ldots, T_{e}\right\}
$$

and,

$$
\lim _{T_{e} \rightarrow+\infty} R_{t}=0
$$

After the insertion of (6) into (5), the first order condition with respect to $R_{t}$ gives

$$
R_{t}=\left[\frac{p_{t}\left(1-\chi_{N R E}\right) \theta}{D_{t}^{\frac{1}{\theta}} V}\right]^{\frac{\theta}{1-\theta}}, \quad \forall t \in\left\{0, \ldots, T_{e}\right\}
$$

\section{Profit maximization of RE producer}

We suppose that a very large (and never binding) flow of renewable primary energy (aggregation of solar radiant energy, geothermal, wave, and tidal energies) is accessible to the economy and that a price-taking representative firm is in charge of its exploitation. In order to capture the annual gross primary renewable energy flow $F_{t}$ some capital $G_{t}$ is obviously necessary and a fraction $\chi_{R E}$ of the gross energy output is self-consumed. Thus, in each period $t$, the RE producer maximizes its profit $\Omega_{t}$ and consequently chooses a capital stock $G_{t}$ in order to deliver the flow $F_{t}\left(1-\chi_{R E}\right)$ of available primary renewable energy sold at the unitary price $p_{t}$ by solving

$$
\max _{F_{t}, G_{t}} \Omega_{t}=\left(1-\chi_{R E}\right) p_{t} F_{t}-v G_{t}, \quad \forall t \in\{0, \ldots, T\}
$$

under constraint,

$$
G_{t}=\left(F_{t} B_{t}\right)^{\frac{1}{\gamma}}, \text { with } 0<\gamma<1 \quad \forall t \in\{0, \ldots, T\}
$$

Where $B_{t}$ represents the capital intensiveness of the RE producer (i.e. the capital requirement per unit of RE output), whose detailed definition is given in section 3.3. The fact that $0<\gamma<1$ means that returns to scale are decreasing and that consequently the capital intensiveness of the RE firm increases with the production level. ${ }^{6}$ Once (11) is injected into (10), the first order condition with respect to $F_{t}$ leads to,

$$
F_{t}=\left[\frac{p_{t}\left(1-\chi_{R E}\right) \gamma}{B_{t^{\frac{1}{\gamma}}}^{\frac{1}{\gamma}}}\right]^{\frac{\gamma}{1-\gamma}}, \quad \forall t \in\{0, \ldots, T\}
$$

6. Dale et al. (2011) used two databases of the National Renewable Energy Laboratory (NREL, 2010a, 2010b) to demonstrate that the frequency of wind and solar power sites in the USA is an inverse function of their productive potential, meaning that over time the availability of optimal sites will decrease. In the same view, Honnery and Moriarty (2009), and Hoogwijk et al. (2004) have shown that as wind energy production increases, the marginal capacity factor of wind turbines decreases. 


\section{Profit maximization of final good producer}

The total primary energy $E_{t}$ available to the final good sector is:

$$
E_{t}=\begin{array}{cc}
R_{t}\left(1-\chi_{N R E}\right)+F_{t}\left(1-\chi_{R E}\right), & \forall t \in\left\{0, \ldots, T_{e}\right\} \\
F_{t}\left(1-\chi_{R E}\right), & \forall t \in\left\{T_{e}+1, \ldots, T\right\} .
\end{array}
$$

This available primary energy $E_{t}$ is combined with capital services $H_{t}$ in a Cobb-Douglas production function in order to produce the final output good $Y_{t}$ representing the macroeconomic product. The formulation of a production function must be independent of the choice of units, hence we introduce the dimensionless variables $y_{t} \equiv \frac{Y_{t}}{Y_{0}}, a_{t} \equiv \frac{A_{t}}{A_{0}}, e_{t} \equiv \frac{E_{t}}{E_{0}}$, and, $h_{t} \equiv \frac{H_{t}}{H_{0}}$, where $Y_{0}, A_{0}, E_{0}$, and $H_{0}$ are given quantities in the initial reference period. Hence,

$$
y[a, e, h]_{t}=\left(a_{t} e_{t}\right)^{\alpha} h_{t}^{1-\alpha}, \quad \forall t \in\{0, \ldots, T\} .
$$

and,

$$
Y_{t}=y[a, e, h]_{t} Y_{0}, \quad \forall t \in\{0, \ldots, T\}
$$

The output elasticities of useful energy and capital services inputs are constant and respectively represented by $\alpha$ and $1-\alpha$. We follow Ayres and War (2009) and other authors such as Cleveland et al. (1984) who have earlier emphasized that the aggregate technology level $A_{t}$ of the economy is formally represented by the efficiency with which primary exergy contained in fossil fuels and renewable energy flows is converted into useful exergy services in the forms of light, heat, electricity, and mechanical drive (i.e. motion). Hence, technological change corresponds to gains in the efficiency of primary-to-useful exergy conversion (i.e. increases of $A_{t}$ ). Formally, the primary-touseful exergy conversion efficiency $A_{t}$ is the product of: (i) the primary-to-final efficiency with which primary exergy contained in fossil fuels and the solar flow is converted in final exergy in the forms of carriers such as liquid fuels (e.g. gasoline), compressed gas, electricity and hightemperature heat; with (ii) the final-to-useful efficiency with which exergy contained in these final forms is converted into useful exergy services in the forms of light, heat, electricity services and mechanical drive. Hence, $A_{t} E_{t}$ represents the useful exergy ${ }^{7}$ provided in the forms of light, heat, electricity services and mechanical drive to the real economy. Of course, another part of the improvement of economic productivity comes from the division and organization of labor, the enhancements of laborer skills, the beneficial effects of inclusive institutions (which, for example, protect private property rights and consequently incentivize innovation and R\&D), and the recent contribution of information and communication technologies. Such attributes are embedded in the labor activated effective capital services $H_{t}$, whose optimal value is found by considering the final good price as the numeraire, and that the representative firm in the final good sector seeks to solve:

7. The term "useful work" is also used in the literature (see Ayres and Warr, 2009; Warr et al., 2010; Warr and Ayres, 2012).

Copyright (C) 2018 by the IAEE. All rights reserved. 


$$
\max _{E_{t} H_{t}} \Psi_{t}=Y_{t}-p_{t} E_{t}-v H_{t}, \quad \forall t \in\{0, \ldots, T\}
$$

under constraint (14) and (15). The resolution of this problem implies to combine the first order conditions with respect to $E_{t}$ and $H_{t}$ in order to find:

$$
H_{t}=\frac{1-\alpha}{\alpha} \frac{p_{t}}{v} E_{t}, \quad \forall t \in\{0, \ldots, T\}
$$

Combining (17) with (14)-(15) in the first order condition with respect to $E_{t}$ gives (after mathematical arrangements):

$$
p_{t}=\alpha \frac{Y_{0}}{H_{0}}\left(\frac{A_{t} H_{0}}{A_{0} E_{0}}\right)^{\alpha}\left(\frac{1-\alpha}{\alpha \nu}\right)\left\{^{-\alpha \frac{1}{\alpha}}, \quad \forall t \in\{0, \ldots, T\}\right.
$$

For the clarity of the following of the presentation let us define now the saving rate of the economy $S_{t}$ as the ratio of investment $I_{t}$ to the macroeconomic product $Y_{t}$ :

$$
S_{t}=\frac{I_{t}}{Y_{t}}, \quad \forall t \in\{0, \ldots, T\}
$$

\subsection{Endogenous Technological Change}

The aggregate technological level $A_{t}$ is necessarily bounded from above by a strictly positive constant $\bar{A}$ representing the maximum efficiency of primary-to-useful exergy conversion that the economy will ultimately reach in the future. This positive upper bound is strictly inferior to one since the second law of thermodynamics imposes that perfect (i.e. 100\%) efficiency of primary-touseful exergy conversion is impossible. ${ }^{8}$ This uncertain parameter (for which we test several values in section 4) is taken as exogenous. The technological level increases over time at speed $\xi_{t}$ and at some point (when the maximum limit $\bar{A}$ is close) the incremental gains in $A_{t}$ are so small that the dynamic system describing the economy is in a quasi-steady state. Hence, with $t_{\Delta A_{t} \max }$ as the particular time at which the growth rate of the technological level (i.e. the technological change) is maximum, we define the law of motion of $A_{t}$ as

$$
A_{t}=\underline{A}+\frac{\bar{A}-\underline{A}}{1+\exp \left(-\xi_{t}\left(t-t_{\Delta A_{t} \max }\right)\right)}, \quad \forall t \in\{0, \ldots, T\} .
$$

8. Ayres and Warr (2009, p.52-53) highlight that technological change at the macro level is ultimately defined by the limiting efficiency of all metallurgical, chemical and electronic processes at micro levels, which in turn depends essentially on the properties of structural materials. Indeed, some technologies, such as prime movers and many metallurgical reduction and synthesis processes, depend on the temperatures, and in some cases, pressures, achievable in a confined space. These are limited by the strength and corrosion resistance (chemical inertness) of structural materials at elevated temperatures. In the same way, turbines efficiencies also depend on the precision with which blades, piston rings, gears and bearings can be manufactured, which depends in turn on the properties of materials being shaped and the properties of the ultra-hard materials used in the cutting and shaping of tools. 
Furthermore, we suppose that the speed of convergence $\xi_{t}$ between the initial technological level $\underline{A}$ and its asymptotic value $\bar{A}$ (verifying $0<\underline{A}<\bar{A}$ ) depends on the variation of the knowledge stock of the economy. This (potentially infinite) knowledge stock (that we do not represent) depends on the effort deployed in the R\&D sector in previous periods that itself follows the saving rate of the economy (i.e. the level of investment compared to the level of economic production) of these same previous periods. In addition, the more recent the saving rate, the higher its influence on $\xi_{t}$. Hence, we define the speed of convergence $\xi_{t}$ of the technological level as the first order exponential smooth of the saving rate of the economy during the $N$ previous periods (where $N$ is defined through calibration). With $\sigma$ as the share of the macroeconomic investment going to $\mathrm{R} \& \mathrm{D}$, we have

$$
\xi_{t}=\begin{array}{cl}
\sigma S_{0} & \text { for } t=0 \\
\sigma\left(\frac{2}{N+1}\right) S_{t-1}+\left(\left\{-\frac{2}{N+1}\right) \xi_{t-1},\right. & \forall t \in\{1, \ldots, T\} .
\end{array}
$$

This formulation of the technological level insures that in our model both technological change and economic growth are endogenous.

\subsection{Unitary Capital Requirements}

\section{Unitary capital requirement in NRE sector}

An accurate formulation of the nonrenewable capital $\operatorname{cost} D_{t}$ should, to our mind, necessarily reproduce three facts: (i) the cost associated with nonrenewable energy extraction must necessarily increase with cumulative production, this is because easier-to-exploit resources are used up first before attention turns to deeper and more remote resources (see Murphy and Hall (2011) for a graphic representation of this fact in the case of oil production) ${ }^{9}$ (ii) the initial unitary cost of NRE production was above the RE production cost before the nineteenth century, this is necessary to explain that despite being known since antiquity, coal was not produced on an industrial scale before wood charcoal became scarce and expensive in England in the late eighteenth century; (iii) learning processes and R\&D have so far allowed a decrease of the NRE production cost. Since we did not find in the literature a formulation that would suit these three prerequisites, the NRE unitary capital requirement proposed in this model is (to the best of our knowledge) unique to the present article.

The capital requirement per output unit of nonrenewable energy, $D_{t}$, is composed of two parts as defined in equation (22) and shown in Figure 1. The first part increases through the extraction process because of the quality depletion of the NRE resource, and the second part decreases through time thanks to learning and R\&D processes. Hence, the first term depends on the ratio of nonrenewable resource depletion $\phi_{t}$, varying between 0 when the nonrenewable energy resource is still virgin and 1 when it is fully depleted. The second term depends on the ratio of technological level advancement, varying between 0 when the aggregate technological level equals its lower bound $\underline{A}$ and 1 when the aggregate technological level equals its upper bound $\bar{A}$. The idea behind this relation is that even though we do not explicitly represent the specific R\&D of the energy sector, we can fairly assume that the different sectors of the economy evolve with technological

9. The rational tendency of humans to first use easier-to-exploit high quality resources before turning towards harderto-exploit lower quality resources is commonly known as the "Best First Principle" (Hall and Klitgaard, 2012). 
Figure 1: Capital Cost per Output Unit of Nonrenewable Energy. This Example is Obtained with $D_{0}=0.3, \tilde{D}=0.2, \omega_{1}=5, \omega_{2}=0.5$, and $\delta=4$

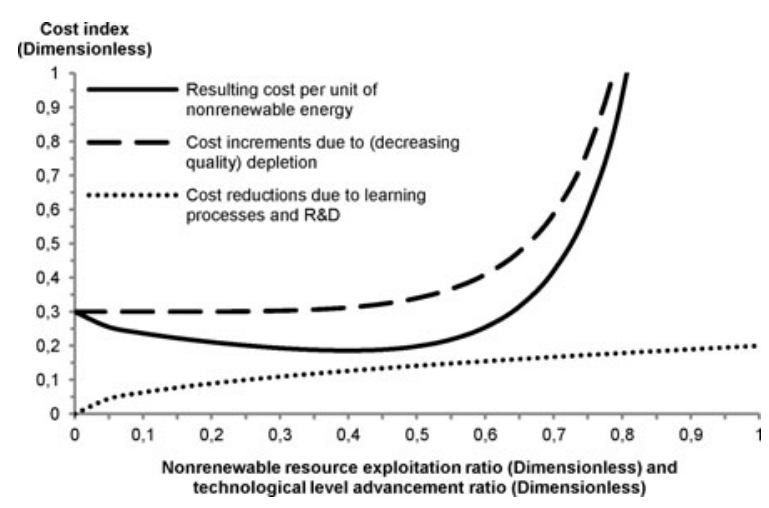

consistency. ${ }^{10}$ Hence, even if from a formal point of view $A_{t}$ represents the efficiency of primaryto-useful exergy conversion in the final good sector, we postulate that this variable, after being normalized between 0 and 1 , is a proxy of the technological level of the energy sector. With $D_{0}$ as the initial capital cost per NRE output unit, $\tilde{D}$ as the maximum capital cost reduction thanks to learning and $R \& D$ processes, and $\delta$ as a constant parameter representing the rate of quality degradation of the NRE resource, we can define $D_{t}$ as

$$
D_{t}\left(\phi_{t}, A_{t}\right)=D_{0} \exp ^{\delta \phi_{t}^{\omega_{1}}}-\tilde{D}\left(\frac{A_{t}-\underline{A}}{\bar{A}-\underline{A}}\right)\left\{, \quad \forall t \in\left\{0, \ldots, T_{e}\right\} .\right.
$$

Where $\omega_{1}$ and $\omega_{2}$ are positive constants determined when calibrating the model on historical global data in section 4 . The exploited resource ratio $\phi_{t}$ is defined as

$$
\phi_{t}=\frac{t_{\text {length }} \sum_{i=0}^{t-1} R_{i}}{\mathcal{R}} \in[0,1], \quad \forall t \in\left\{0, \ldots, T_{e}\right\}
$$

\section{Unitary capital requirement in RE sector}

To be accurate, the capital requirement per renewable energy output unit, $B_{t}$, should be represented by a decreasing function since, over time, less capital is necessary to capture the same amount of primary renewable energy thanks to learning processes and R\&D. Furthermore, as for the NRE sector, we postulate that the RE sector is technologically consistent with the rest of the

10. Of course, some sectors might have faster technological improvements than others, and that is particularly true regarding the distinction we make between the two primary energy-producing sectors on the one hand, and the final good energy-consuming sector on the other. Nevertheless, we think that in average such sectoral discrepancies in technological levels cannot last more than a few decades. Indeed, on a larger time horizon, technological level gaps between sectors would imply investment opportunities and subsequent reallocation of financial capital and hence R\&D. Considering that our time step is twenty years, we think that postulating a technological consistency between the different sectors of our model is rather justified. 
economy, so that $B_{t}$ is a function of $A_{t}$. The sigmoid decreasing function $B_{t}\left(A_{t}\right)$ describing the capital cost per unit of renewable energy output starts at value $\bar{B}$ and decreases at a constant speed $\tau>0$ to a strictly positive bound $\underline{B}$ since the production of any RE flow would always require a minimum quantity of capital:

$$
B_{t}\left(A_{t}\right)=\bar{B}-\frac{\bar{B}-\underline{B}}{1+\exp \left(-\tau\left(A_{t}-A_{\Delta B_{t} \max }\right)\right)}, \quad \forall t \in\{0, \ldots, T\} .
$$

Where $A_{\Delta B_{t} \max }$ is the particular technological level at which the function $B_{t}$ presents an inflexion point (i.e. the rate of degrowth of $B_{t}$ is maximum when $A_{t}=A_{\Delta B_{t} \max }$ ). In addition, we suppose that the final unitary cost of renewable energy production $\underline{B}$ also depends on the final technological level $\bar{A}$ of the final good sector. Precisely, the higher the ratio of ultimate technological level gain $\bar{A} / \underline{A}$, the lower the final unitary cost of RE production $\underline{B}$ should be compared to its initial value $\bar{B}$. Hence, with $\eta$ as a parameter found through calibration to historical data (see section 4), we suppose

$$
\underline{B}=\frac{\bar{B}}{(\bar{A} / \underline{A})^{\eta}}, \text { with } 0<\eta<1 \text {. }
$$

\subsection{EROI of Energy Sectors}

In order to define the energy-return-on-investment (EROI) of the two energy sectors, we need to breakdown the saving rate $S_{t}$ in three parts $S_{H, t}, S_{Z, t}, S_{G, t}$ defined respectively as the fraction of the economic output of period $t$ invested in period $t+1$ in the final good sector, NRE sector, and RE sector respectively:

$$
S_{t}=S_{H, t}+S_{Z, t}+S_{G, t}, \quad \text { with } S_{H, t}=\frac{H_{t+1}}{\lambda Y_{t}}, \quad S_{Z, t}=\frac{Z_{t+1}}{\lambda Y_{t}}, \text { and } S_{G, t}=\frac{G_{t+1}}{\lambda Y_{t}}
$$

According to Hall et al. (2014), the EROI is "the ratio between the energy delivered by a particular fuel to society and the energy invested in the capture and delivery of this energy". King et al. (2015) point out that this definition is rather loose and that a clear distinction should be made between yearly power return ratios (PRRs) of annual energy flows and energy return ratios (ERRs) of full life cycle energy systems (i.e. cumulated energy production divided by total energy invested) which more formally represent EROIs. Understandably, energy return ratios represent integrals of power return ratios over the entire life cycle of the energy system under consideration. Recalling that in our theoretical model defined in discrete times, the time length between two consecutive periods equals the capital services lifetime, PRRs and ERRs are exactly the same in our particular theoretical setting. Furthermore, PRRs and ERRs can differ regarding the system boundary of their energy output (numerator) and energy input (denominator). We will consider as energy outputs the production levels of gross primary energy $R_{t}$ or $F_{t}$ in the NRE or RE cases respectively. The invested energy takes usually two forms: direct energy inputs in the form of self-consumption and external energy investments (generally as final carriers like electricity or liquid fuels), and indirect inputs energy embodied in capital and services. Since the energy sectors of the model represent upstream sectors producing primary energy, and considering that we do not represent downstream sectors that convert primary energy in final forms, the direct energy inputs of the two primary energy 
producing sectors are only represented by their respective self-consumptions. In order to calculate the indirect energy investments embodied in capital services, let us consider the example of the nonrenewable sector in which the production of the gross primary energy output $R_{t}$ requires the capital stock level $Z_{t}$ that comes from the fraction $S_{Z, t-1}$ of economic output $Y_{t-1}$. Since the production of $Y_{t-1}$ has required the consumption of the primary energy $E_{t-1}$, it follows that the quantity of indirect energy embodied in the NRE sector capital services used in period $t$ is $S_{Z, t-1} E_{t-1}$. Finally, given all previous precisions and referring to King et al. (2015) definitions, the EROI (a denomination we keep for convenience) we compute is formally a gross power ratio that due to our discrete time setting equals its integral over time (i.e. gross energy ratio ). For the NRE sector, the $E R O I_{N R E, t}$ is defined as:

$$
E R O I_{N R E, t}=\frac{R_{t}}{R_{t} \chi_{N R E}+S_{Z, t-1} E_{t-1}} .
$$

Similarly, the $E R O I_{R E, t}$ of the RE production in period $t$ is:

$$
E R O I_{R E, t}=\frac{F_{t}}{F_{t} \chi_{R E}+S_{G, t-1} E_{t-1}} .
$$

Finally, it is possible to define the EROI of the whole primary energy sector since delivering the total gross primary energy $R_{t}+F_{t}$ to the final sector has directly required the self-consumption $R_{t} \chi_{N R E}$ and $F_{t} \chi_{R E}$, and indirectly required the embodied energy $S_{Z, t-1} E_{t-1}+S_{G, t-1} E_{t-1}$. Thus globally, the $\mathrm{EROI}_{t}$ of the entire energy sector in period $t$ is:

$$
E R O I_{t}=\frac{R_{t}+F_{t}}{R_{t} \chi_{N R E}+F_{t} \chi_{R E}+\left(S_{Z, t-1}+S_{G, t-1}\right) E_{t-1}} .
$$

Due to the highly nonlinear formulation of our model, studying its potential analytical solution would prove to be rather difficult if not impossible. Thus, it is preferable to study its dynamics through simulation. In the coming section 4 , the model is calibrated on global historical data in order to reproduce the last two hundred and sixty-five years or so in terms of energy supply, technological improvement, and gross world product (GWP). We also study in this section the future transition towards complete renewable energy, which the global economy will have to accomplish because of the finite nature of fossil fuels and nuclear energy, and we assess in which circumstances this transition could have negative impacts on the GWP pattern.

\section{CALIBRATION AND SIMULATION OF THE MODEL}

\subsection{Global Historical Data, Parameters, and Scenarios}

\section{Global historical data}

Four time series are used to calibrate the model on global historical data: nonrenewable primary exergy production, renewable primary exergy production, efficiency of primary-to-useful exergy conversion, and GWP. Since the time period $t_{\text {length }}$ between $t$ and $t+1$ corresponds to the average capital lifetime set to twenty years, our data time series consist of fourteen discrete points 
from $1750(t=0)$ to $2010(t=13)$. Of course, the different references used to retrieve global historical data do not always propose values for the specific year we need. Hence, the 20-years interval estimations of global historical data reported in Table A1 of the Appendix are rounded up values (comparison with original data of respective references are provided in Figure A1 of the Appendix).

To suit the model structure we have aggregated in a single NRE production the different historical data for global primary production of coal, oil, gas, and nuclear energy. Following Kümmel (2011) we made the assumption that these primary energy forms expressed in exajoule per year (EJ/yr) represent $100 \%$ exergy. Energy production values have been retrieved through the online data portal of The Shift Project (2015), which is built on the original work of Etemad and Luciani (1991) for the 1900-1980 time period and EIA (2014) for 1981-2010. Prior to 1900, we have completed the different fossil fuel time series with the original 5-years interval data of Etemad and Luciani (1991) and filled the gaps using linear interpolation. In the same way, the historical global primary production of biomass energy (woodfuel and crop residues ${ }^{11}$ ) from Fernandes et al. (2007) and Smil (2010) were aggregated with the historical global renewable energy production of The Shift Project (2015) for hydro, wind, solar, geothermal, wastes, ocean (wave, tidal, OTEC), and modern biofuels into a single primary renewable energy production expressed in EJ/yr.

We give in Table A1 an estimation of the efficiency of primary-to-useful exergy conversion from 1750 to 2010 at global scale. It is important to emphasize that this estimate does not come from any calculation but only represents a best guess after considering the work of Warr et al. (2010) who have estimated the efficiency of primary-to-useful exergy conversion for the US, the UK, Japan, and Austria from 1900 to 2000 as shown in Figure A1b.

Regarding the gross world product (GWP) expressed in Billion 1990 International GearyKhamis dollar, ${ }^{12}$ we use the data of Maddison (2007) from 1750 to 1949 and the GWP per capita of The Maddison Project (2013) multiplied by the United Nations (2015) estimates of global population from 1950 to 2010 .

\section{Parameters and scenarios}

All simulations are performed up to the time horizon $T=25$ corresponding to the year 2250. The initial technological level is logically set to $A_{0}=\underline{A}=0.025$ and we can also define $Y_{0}=$ 435 Billion G-K. $\$ 1990$ from Table A1. Parameters $\chi_{N R E}$ and $\chi_{R E}$ are arbitrarily set equal to 0.01 because with have no reliable data to choose otherwise. All other parameters values synthesized for clarity in Table A2 of the Appendix are necessarily found through the calibration of the model to historical data. We have performed such procedures with two prerogatives: (i) the calibration must remain robust under the different scenarios that are tested; (ii) the scenarios must differ by the least possible number of differences in parameter values. Logically we found that the main determinant of a given scenario is the ultimate value $\bar{A}$ towards which technological level $A_{t}$ converges. As shown in Figure A1b of the Appendix it can be fairly assessed that the global technological level $A_{t}$ has evolved from 0.025 in 1750 to 0.125 in 2010 . Since the maximum attainability of the efficiency of primary-to-useful exergy conversion is necessarily below 1 , we have tested

11. Formally, food provided to laborers and fodder supplied to draft animals should be added to traditional biomass energy estimates, but it is generally discarded due to difficulties of estimation. This is also the case for traditional windmills and water wheels.

12. The 1990 International Geary-Khamis dollar (Int. G-K. \$1990), more commonly known as the international dollar, is a standardized and fictive unit of currency that has the same purchasing power parity as the U.S. dollar had in the United States in 1990. 
Table 1: Specific Parameters Values of Low, Medium, High, and Extra-High Scenarios

\begin{tabular}{|c|c|c|c|c|c|}
\hline Parameter & Definition (unit) & Low & Medium & High & Extra-High \\
\hline $\bar{A}$ & $\begin{array}{l}\text { Final technological level of the economy, i.e. final efficiency } \\
\text { of primary-to-useful exergy conversion in the final good } \\
\text { sector (dmnl). }\end{array}$ & 0.25 & 0.35 & 0.45 & 0.65 \\
\hline$t_{\Delta A_{t} \max }$ & $\begin{array}{l}\text { Time of maximum technological change (model time period/ } \\
\text { actual year). }\end{array}$ & $\begin{array}{l}13.35 \\
(2017)\end{array}$ & $\begin{array}{l}14.45 \\
(2039)\end{array}$ & $\begin{array}{l}15.15 \\
(2053)\end{array}$ & $\begin{array}{c}16.0 \\
(2070)\end{array}$ \\
\hline$\tilde{D}$ & $\begin{array}{l}\text { Maximum capital cost reduction per unit of nonrenewable } \\
\text { energy thanks to learning processes and } R \& D(B \$ / E J) \text {. }\end{array}$ & 6.180 & 6.295 & 6.365 & 6.458 \\
\hline
\end{tabular}

several values between 0.15 and 0.95 and decided to present the results for four scenarios, respectively described by the following $\bar{A}$ values: $0.25,0.35,0.45$ and 0.65 . Once $\bar{A}$ is defined, we found that in order to respect the objectives (i) and (ii) previously cited, only two additional parameters needed to be tuned, namely $\tilde{D}$ and $t_{\Delta A_{t} \max }$. Hence, the four different scenarios, called Low, Medium, High, and Extra-High, are exactly determined by their common parameters synthetized in Table A2, and their specific parameters presented in Table 1 .

One important parameter of the model merits specific attention: the ultimately recoverable resource (URR) $)^{13}$ of nonrenewable energy, $\mathcal{R}$. This parameter represents the total amount of nonrenewable energy that may be recovered at positive net energy yield, i.e. at EROI greater or equal to unity. A literature review led to the choice of McGlade and Ekins (2015) estimations for coal, oil, and gas URR, and the IIASA (2012) estimate for uranium URR. As reported in Table A3 of the Appendix, the conversion in exajoule (EJ) and aggregation of these different estimates yields a global nonrenewable URR estimate $\mathcal{R}=175,500$ EJ. Contrary to what one might think, sensitivity analyses of the model to this parameter (not presented in the paper for the sack of brevity) have shown a great robustness of its qualitative results. Changing the value of $\mathcal{R}$ does not change the dynamics of the model because it is necessarily balanced by a change in other parameter values (in particular $D_{0}, \omega_{1}$, and $\omega_{2}$ ) in order for the calibration to the historical data to remain valid.

\subsection{Simulation Results}

Figure 2 summarizes the simulation results of the most interesting variables of the model (primary energy productions, technological change, and GWP) for the entire 1750-2250 time frame. Figure A2 of the Appendix shows the same data on the restricted 1750-2010 time frame in order to better observe the calibration adequacy of the model to historical data.

\section{Calibration results}

Figure A2 of the Appendix shows that the global historical patterns of nonrenewable and energy productions (A2ab), technological change (A2c), and GWP (A2d) are acceptably reproduced

13. According to British Petroleum (2015): the "URR is an estimate of the total amount of a given resource that will ever be recovered and produced. It is a subjective estimate in the face of only partial information. Whilst some consider URR to be fixed by geology and the laws of physics, in practice estimates of URR continue to be increased as knowledge grows, technology advances and economics change. The ultimately recoverable resource is typically broken down into three main categories: cumulative production, discovered reserves and undiscovered resource." On the other hand, Sorrell et al. (2010) highlight that unlike reserves, URR estimates are not dependent on technology assumptions and thus should only be determined by geologic hypotheses. Unfortunately, this apparent contradiction of the URR definition is only a tiny example of the fuzziness of points of view that one could find in the literature regarding the different notions of nonrenewable resources and reserves. 
Figure 2: Historical vs. Simulated Data, 1750-2250: (a) Primary Nonrenewable Energy Production, (b) Primary Renewable Energy Production, (c) Global Efficiency of Primary-to-useful Exergy Conversion, (d) Gross World Product
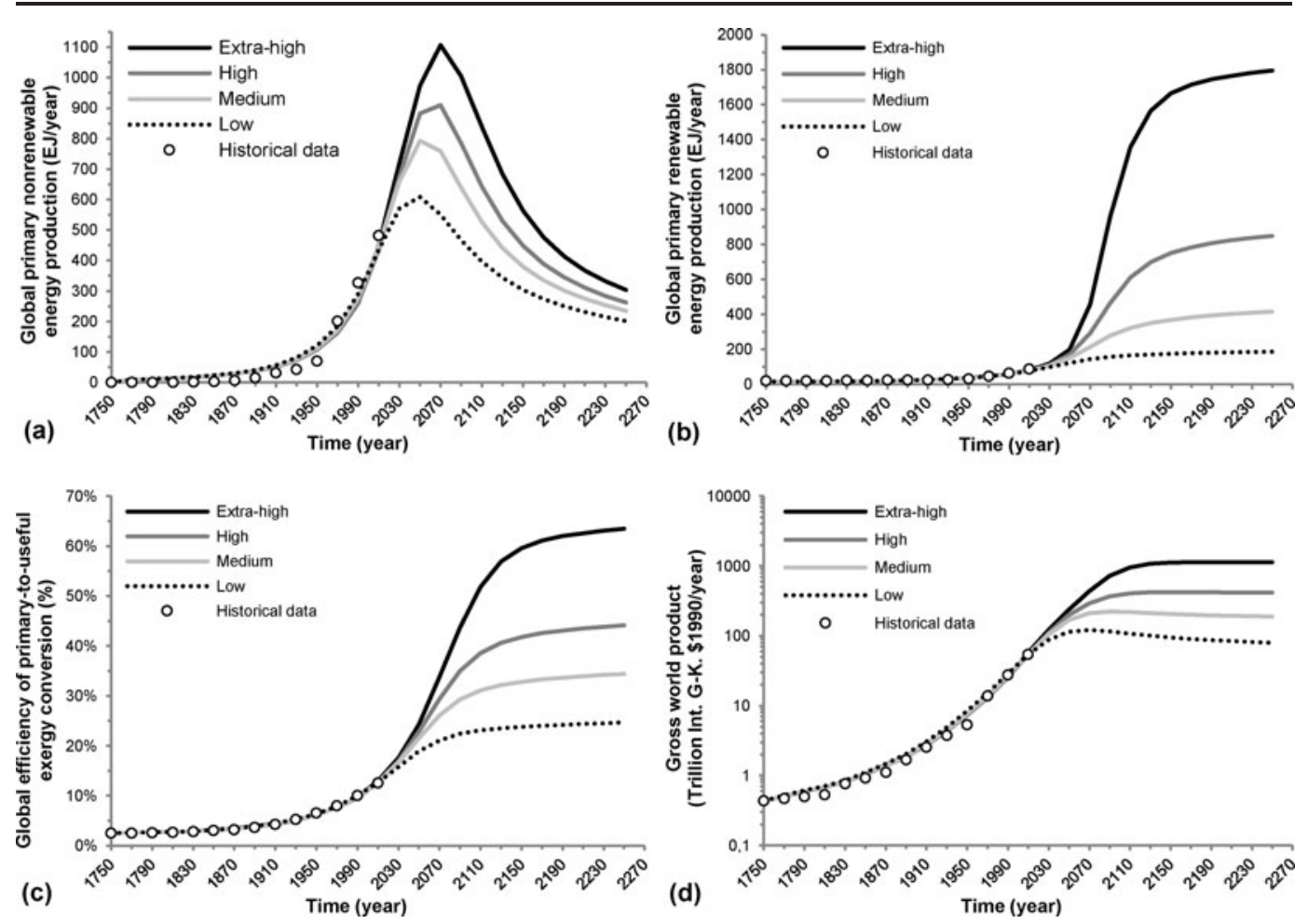

by the model. The model calibration is particularly good for past global efficiency of primary-touseful exergy conversion and for past GWP. Regarding nonrenewable energy, the model slightly overestimates the historical global trend up to 1950. Concerning the global production of renewable energy (for which data uncertainty is higher than for nonrenewable energy), we have not been able to reproduce the nearly stagnant trend between 1750 and 1910 as the model is only capable of producing continuously increasing dynamics for this variable.

\section{Prospective results}

When the model is simulated up to 2250 , differences between scenarios clearly appear. Obviously, this is visible in Figure 2c where the simulated values of the global efficiency of primaryto-useful exergy conversion are presented for the four scenarios. As formalized in the model, the technological change dynamics directly influence the nonrenewable and renewable energy productions paths respectively presented in Figure $2 \mathrm{a}$ and $2 \mathrm{~b}$ for the four scenarios. Concerning nonrenewable energy, the higher the final technological level, the higher the value of the production peak, and possibly the higher the time of that peak (2050 for the Low and Medium scenarios, 2070 for the High and Extra-high scenarios). Regarding the renewable energy production, its final value is obviously higher if the final technological level is higher. As can be seen in Figure $2 d$ where the 
GWP is expressed on a log scale for convenience, the energy supply dynamics has a great impact on the economic production. The final level of renewable energy production primarily determines the final GWP level, but more interestingly the combined dynamics of the nonrenewable and renewable energy productions, i.e. the time path of the energy transition, determine the more or less smoothed course of the GWP. More precisely, if the nonrenewable energy peak is too high compared to the final combination of renewable production and technological level (as in the Low and Medium scenarios), the GWP can peak and then decrease before stabilizing (the log scale of Figure $2 \mathrm{~d}$ hide this important result of the model, which is more visible in the Figure $5 \mathrm{~d} 1 / \mathrm{d} 2$ of section 5).

It is important to be clear here: the negative GWP patterns (overshoot before degrowth) of the Low and Medium scenarios do not arise solely because their final technological levels $\bar{A}$ (respectively at 0.25 and 0.35 ) are too low in absolute terms. Rather, the negative impact of the energy transition on economic growth is due in our model to the final value of the technological level and the way this variable influence the production cost of the two energy forms. Recall that this link between the technological level $A_{t}$ of the final good sector and the production costs $D_{t}\left(A_{t}, \phi_{t}\right)$ and $B_{t}\left(A_{t}\right)$, of the nonrenewable and renewable energy sectors respectively, was established to ensure a technological consistency across all sectors of the model. No matter how one would like to change in the parameter values of the model, the negative impact of the energy transition on economic growth observed for the Low and Medium scenarios is unavoidable. In the coming section 5 we discuss different strategies that can help smoothing the GWP dynamics in scenarios that use the Low scenario settings in terms of parameter values. Before turning to this section, it is worth analyzing the dynamics of the EROI of the nonrenewable and renewable energy productions since, apart from Fagnart and Germain (2014), our model is the first to introduce these crucial variables in a neoclassical framework.

\section{EROI of energy sectors}

When computing the EROIs of nonrenewable and renewable energy production, we found quite strange results in light of the EROI literature. Raising these issues is important in order to indicate the features of our model that should deserve particular attention in order to be improved in future research. First, within a given scenario and a given time period, EROIs of nonrenewable and renewable energy productions have exactly the same value, i.e. $E R O I_{N R E, t}=E R O I_{R E, t}=$ $E R O I_{t}$. This outcome comes from two particular features of our model: (i) NRE and RE productions are perfect substitutes since they are sold at the same price, (ii) both productions have the same level of self-consumption since we have assumed $\chi_{N R E}=\chi_{R E}$. The first hypothesis is a modeling choice (and we think that including two different prices would not be as simple as one might think because the model would need further complexification in order to remain closed), the second hypothesis is due to the absence of reliable data and therefore the option to choose otherwise.

Second, as shown in Figure 3, the EROIs of the economy have relatively low and restricted values (always between 4.1 and 5.8). These low values of the simulated EROIs might surprise people accustomed to the EROI literature. Indeed, generally accepted orders of magnitude of EROI are around 8-10 for traditional biomass energy (woodfuel, crop residues), 1-2 for modern biofuels, 4-20 for modern renewables (wind, solar, etc.), 10-40 for conventional oil, 40-60 for gas, and 60100 for coal (Hall et al., 2014). These estimates generally include direct energy consumption in the form of final energy (electricity, liquid fuels, etc.) and indirect energy embodied in physical capital. One should not forget that in our model, the quantity of capital services $Z_{t}$ and $G_{t}$ formally represent labor activated effective capital services or, in other words, the aggregated output of physical capital, 
Figure 3: EROI of the Global Economy, 1770-2250

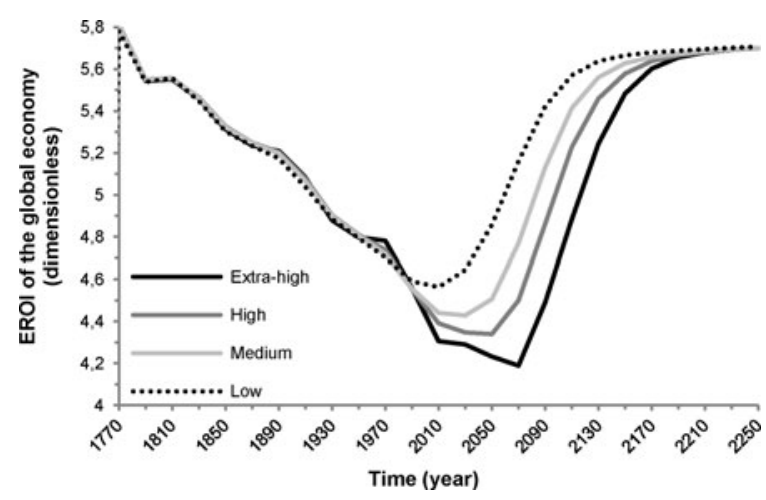

routine labor and human capital. ${ }^{14}$ Hence, in our model the denominator of the EROI not only includes the energy embodied in physical capital formation but also the energy necessary to sustain labor (i.e. to provide, at a minimum, food and shelter to workers), and to support their skills development. As a consequence, the resulting EROIs of the model represent full lifecycle energy ratios of primary energy production and are thus necessarily quite low compared to conventional values found in the literature that do not take into account such an extended input boundary.

Third, the simulated EROIs have clear U-shapes over the entire time frame, whereas the EROI theoretical model of Dale et al. (2011) suggest just the opposite. We interpret those U-shapes as the mark of the technological influence in the final good sector. Once the technological level $A_{t}$ takes off, producing the final output good $Y_{t}$ require less and less primary energy, in other words, the energy embodied in capital services decreases. Hence, even if the capital intensiveness of both energy-producing sectors increases due to decreasing marginal returns, their EROI increase after reaching a minimum because the energy embodied in each unit of capital decreases. Logically, when the technological level $A_{t}$ approaches its asymptotic maximal value, the EROI stabilizes. In order to correct this unrealistic feature of our model, it would be necessary to add another sector and to make a clear distinction between the production of an intermediary capital product and the final good product.

Now that the model results have been analyzed, we can turn our attention on the strategies to avoid the unanticipated nonrenewable energy peak and associated renewable energy supply delay, which cause an overshoot and then degrowth of the economic production in the Low and Medium scenarios. At first approximation, this energy lock-in which generates this unfortunate GWP dynamics can be thought of as a failure of the price system to incentive early renewable energy production.

\section{DISCUSSION ON THE IMPLEMENTATION OF A CARBON PRICE}

We consider that the final technological level $\bar{A}$ of the economy, by far the most important parameter determining the dynamics of the model, can hardly be changed endogenously by a given

14. The fact that capital services $Z_{t}, G_{t}$, and $H_{t}$ represent far more than just physical capital also logically translate in the values of the saving rate $S_{t}$ varying between 0.5 and 0.8 for all scenarios, which is indeed pretty high compared to real global saving rates of about 0.22-0.24 (World Bank, 2016).

Copyright (C) 2018 by the IAEE. All rights reserved. 
policy action. This asymptotic value $\bar{A}$ cannot be known a priori but only a posteriori once thermodynamic limits are reached for all the different energy-using devices of the economy, including the ones that have yet to be invented. But the intuition we want to test is that even if this parameter is primordial for determining the ultimate state of the economic system, there must be ways to change the trajectory that leads to this deterministic end. This point is especially important if this path is believed to generate welfare losses because of the (imposed and not chosen) economic degrowth as in the Low and Medium scenarios. In other words, the policy actions that must be investigated are the ones that help avoid as much as possible the lock-in phenomenon described previously that is characteristic of both the real world and our model: the tendency of the economic system to stay accustomed to fossil fuels without anticipating their inevitable supply peak and decline, and the need associated to an early increase of the renewable energy production. Starting from a Low scenario setting in terms of parameter values, the strategy we propose to avoid its adverse outcome (GWP peak followed by a degrowth phase) is to implement a tax on the nonrenewable energy production and to use the income revenue from this tax to direct the energy transition dynamics and smooth its negative impact on GWP. Such a tax could be indexed to the polluting potential of the fossil energy and more precisely to its greenhouse gases (GHG) content (abstracting from the fact that the nonrenewable resource of the model also contain some GHGfree uranium energy).

Hence, in our model the price that we will exogenously impose on the NRE production could clearly be seen as a carbon price/tax. It is important to understand that the income from the carbon pricing can be used in three different ways that can be combined in various proportions to generate many different policy mixes. Indeed, the annual income from the carbon tax could be used to: (i) subsidize the general $\mathrm{R} \& \mathrm{D}$ sector of the economy in order to accelerate the convergence of $A_{t}$ towards $\bar{A}$; (ii) subsidize the $\mathrm{R} \& \mathrm{D}$ that is specific to the RE sector in order to accelerate the decrease of $B_{t}$ towards $\underline{B}$; or (iii) subsidize a direct increase in the capital investment $G_{t}$ in the RE sector. In the following of this section we will first present the different equation changes resulting from the implementation of the carbon price. Then, the specific mathematical formalization of the uses of the carbon tax income will be successively presented. Finally, we will propose four policy mix scenarios and compare the results of their simulations.

\subsection{Common Equation Changes Due to the Carbon Price Implementation}

Let us define $q_{t}$ as the unitary carbon price at period $t$ (i.e. the carbon price per unit of pollution, hence expressed in $\$ / \mathrm{tCO} 2 \mathrm{eq}$, or $\mathrm{B} \$ / \mathrm{GtCO} 2 \mathrm{eq}$ in order to be consistent with the previous sections). This carbon price is zero prior to the time period $t_{q \text { start }}$ in which it is implemented, and it evolves towards the maximum unitary carbon tax value $\bar{q}$ at exogenous speed $\rho$ following a sigmoid increasing form (Figure 4). The maximum growth rate of the unitary carbon tax occurs when $t=t_{q \text { start }}+t_{q \text { lag }}$, so finally:

$$
q_{t}=\frac{\bar{q}}{1+\exp \left(-\rho\left(t-t_{q \text { start }}-t_{q \text { lag }}\right)\right)}, \quad \forall t \geq t_{q \text { start }}
$$

Since the NRE producer has to pay the price $q_{t}$ for every unit of pollution (B $\$ / \mathrm{GtCO} 2 \mathrm{eq}$ ), he has to pay the amount $q_{t} \kappa$ per unit of nonrenewable energy produced (B $\left.\$ / E J\right)$, with $\kappa$ representing the GHG emission factor of nonrenewable energy (expressed in $\mathrm{GtCO} 2 \mathrm{eq} / \mathrm{EJ}$ ). Hence, we deduce that the annual carbon tax income $Q_{t}$ is defined by: 
Figure 4: Profiles of the Two Possible Carbon Prices $q$ and $q^{\prime}, 2010-2250$

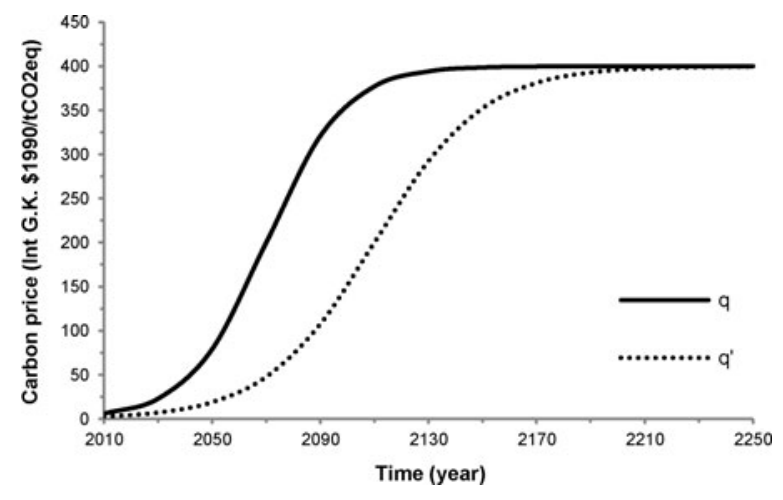

$$
Q_{t}=R_{t} q_{t} \kappa, \quad \forall t \geq t_{q \text { start }}
$$

Implementing the carbon price also logically changes the equations relative to the NRE producer behavior. More precisely, the implementation of the carbon price leads to the replacement of equations (5) and (9) with (32) and (33).

$$
\begin{aligned}
& \max _{R_{t}, Z_{t}} \Pi_{t}=\left(1-\chi_{N R E}\right)\left(p_{t}-q_{t} \kappa\right) R_{t}-v Z_{t}, \quad \forall t \in\left\{0, \ldots, T_{e}\right\} \\
& R_{t}={\frac{\left(1-\chi_{N R E}\right)\left(p_{t}-q_{t} \kappa\right) \theta}{D_{t}^{\frac{1}{\theta}} v}}^{\frac{\theta}{1-\theta}}, \quad \forall t \in\left\{0, \ldots, T_{e}\right\}
\end{aligned}
$$

In addition to the equation changes that concern the NRE producer previously presented, some equation changes will also be specific to each way of using the carbon tax income $Q_{t}$.

\subsection{Specific Equations Change due to Particular Use of the Carbon Tax Income}

Since we have potentially three simultaneous ways to use the carbon tax income, each option represents a share $\beta_{i}, i \in[1,2,3]$ of the total carbon tax income, with $\beta_{1}+\beta_{2}+\beta_{3}=1$.

Option (i): carbon price income used to subsidize the general $R \& D$ sector

A first option is to allocate the carbon tax income to the general $\mathrm{R} \& \mathrm{D}$ sector in order to increase the growth rate $\xi_{t}$ of the technological level. Doing so has a direct effect on the GWP through (14), and an indirect effect through the impact on the nonrenewable and renewable energy production dynamics with (18), (22), and (24). In order to formalize the use of the income from the carbon tax to subsidize the general $R \& D$ sector, we have to replace equation (21) defining the speed of convergence $\xi_{t}$ of the aggregate technological level with the following equation (34):

$$
\begin{array}{cl}
\sigma S_{0}+\beta_{1} \varepsilon_{1} Q_{t-1} & \text { for } t=0 \\
\sigma\left(\frac{2}{N+1}\right) S_{t-1}+\left(\left\{-\frac{2}{N+1}\right) \xi_{t-1}+\beta_{1} \varepsilon_{1} Q_{t-1},\right. & \forall t \in\{1, \ldots, T\} .
\end{array}
$$


Where $\varepsilon_{1}$ measures the efficiency with which the general R\&D sector uses the carbon tax income to produce innovations that materialize in the form of $\xi_{t}$ increases. The functional form given in (34) insures that the higher the carbon tax income of period $t-1$ and the higher the share $\beta_{1}$ of this income dedicated to the general R\&D sector, the faster the technological level will converge towards its upper bound $\bar{A}$.

Option (ii): carbon price income used to subsidize the specific $R \& D$ of the RE sector

A second way to use the income from the carbon tax is to allocate it to the $R \& D$ that is specifically dedicated to the renewable energy sector. Doing so should affect the rate of degrowth of the unitary capital cost of RE production $B_{t}$ towards its lower bound $\underline{B}$. An appropriate way to formalize this is to replace (24) with the following (35):

$$
B_{t}\left(A_{t}\right)=\bar{B}-\frac{\bar{B}-\underline{B}}{1+\exp \left(-\left(\tau+\beta_{2} \varepsilon_{2} Q_{t-1}\right)\left(A_{t}-A_{\Delta B_{t} \max }\right)\right.}, \quad \forall t \in\{0, \ldots, T\}
$$

Where $\varepsilon_{2}$ measures the efficiency with which the specific R\&D of the RE sector uses the carbon tax income to produce innovations that materialize in the form of RE production cost decreases. The functional form given in (35) insures that the higher the carbon tax income of period $t-1$ and the higher the share $\beta_{2}$ of this income dedicated to the specific R\&D of the RE sector, the faster the unitary capital cost of RE production will converge towards its lower limit $\underline{B}$.

Option (iii): carbon price income used as a direct capital investment in the RE sector

The third option for using the income from the carbon tax consists of a direct subsidy to the renewable energy sector in order to increase the amount of available energy-capturing capital. This should be seen as the capacity of the RE producer to install an additional amount of physical capital and hire workers thanks to a subsidy from the carbon tax income of the previous period. To formalize this effect we propose to replace (11) with the following equation:

$$
G_{t}=\left(F_{t} B_{t}\right)^{\frac{1}{\gamma}}+\beta_{3} \varepsilon_{3} Q_{t-1}, \quad \forall t \in\{0, \ldots, T\}
$$

Where $\varepsilon_{3}$ measures the efficiency with which the RE sector uses the subsidy that is received in the previous period to build new capital and hire additional workers in the RE sector. The functional form given in (36) insures that the higher the carbon tax income of period $t-1$ and the higher the share $\beta_{3}$, the higher the additional renewable energy produced in period $t$.

\subsection{Policy Mix Definition, Carbon Price Profile, and Simulations}

\section{Defining policy mix scenarios and carbon price profiles}

Among an infinity of possibilities, we define four different policy mix scenarios characterized by their relative parameters share $\beta_{i}, i \in[1,2,3]$ :

- General $R \& D$ scenario: the totality of the carbon tax income is allocated to the general $R \& D$ sector, so $\beta_{1}=1, \beta_{2}=0$, and $\beta_{3}=0$. 
Table 2: Values for Parameters Defining the Two Possible Carbon Taxes $q$ and $q^{\prime}$

\begin{tabular}{|c|c|c|c|}
\hline Parameter & Definition (unit) & Value for carbon $\operatorname{tax} q$ & Value for carbon tax $q$ ' \\
\hline $\bar{q}$ & $\begin{array}{l}\text { Maximum level of the carbon tax (Int. G-K. } \\
\$ 1990 / \mathrm{tCO}_{2} \mathrm{eq} \text { ) }\end{array}$ & 400 & 400 \\
\hline$\rho$ & Exogenous growth rate of the carbon tax (dmnl) & 1.4 & 1.0 \\
\hline$t_{q \text { start }}$ & $\begin{array}{l}\text { Time period for implementing the carbon tax } \\
\text { (time period) }\end{array}$ & 13 & 13 \\
\hline$t_{q \text { lag }}$ & $\begin{array}{l}\text { Time lag to obtain the maximum rate of growth } \\
\text { of the carbon tax after its implementation time } \\
\text { (time period) }\end{array}$ & 3 & 5 \\
\hline
\end{tabular}

- One third each scenario: the income from the carbon tax is split equally between the three ways of revenue recycling, so $\beta_{1}=1 / 3, \beta_{2}=1 / 3$, and $\beta_{3}=1 / 3$.

- 50/50 RE R\&D/investment scenario: the carbon tax income is split equally between the specific $R \& D$ of the RE sector and the direct capital investment in the RE sector, there is no additional subsidy to the general R\&D sector, so $\beta_{1}=0, \beta_{2}=0.5$, and $\beta_{3}=0.5$.

- 30/70 RE R\&D/investment scenario: $30 \%$ of the income from the carbon tax goes to the specific R\&D of the RE sector, and $70 \%$ is used as a direct capital investment in the RE sector. In this scenario also there is no additional subsidy to the general $\mathrm{R} \& \mathrm{D}$ sector, so $\beta_{1}=0, \beta_{2}=0.3$, and $\beta_{3}=0.7$.

We have chosen to test two exogenous carbon price profiles called $q$ and $q^{\prime}$. They are defined in Table 2 by their respective parameters $\bar{q}, \rho, t_{q \text { start }}$, and $t_{q \text { lag }}$ and shown in Figure 4 . As previously mentioned we make the hypothesis that all new scenarios in which we implement the carbon price start with the parameter settings of the Low scenario. The value of parameter $\kappa$ representing the GHG emission factor of nonrenewable energy is set to $0.085 \mathrm{GtCO} 2 \mathrm{eq} / \mathrm{EJ}$. It is the average value found when dividing the historical global GHG emissions from fossil fuels estimated by Boden et al. (2013) by the historical nonrenewable energy production presented in Table A1. We consider that $\varepsilon_{3}$ has the same value as $\lambda$ since both parameters represent productivities of the transformation of investments goods into productive capital, and that there is no apparent reason to think that they should differ from one sector of the economy to another. Hence, $\varepsilon_{3}=\lambda=7.25$. On the other hand, since we have no clear way to estimate parameters $\varepsilon_{1}$ and $\varepsilon_{2}$, we have arbitrarily chosen the same value of 0.0002 for both parameters, which we found when performing the simulations.

\section{Simulation results of carbon price scenarios}

In Figure 5, we compare the nonrenewable and renewable energy productions, and the GWP of the baseline Low scenario with the four scenarios that include the carbon tax $q$ (left side) or $q^{\prime}$ (right side). Simulations of carbon price scenarios deliver four results. (i) The desired smoothing dynamics of the GWP is only obtained with the 50/50 or 30/70 scenarios in which the carbon tax income is allocated to the specific R\&D of the renewable energy sector and to direct capital investment in renewable energy technologies. (ii) For all scenarios, the GWP smoothing is higher with the more initially stringent carbon price $q$ than with $q$ '. (iii) For the General R\&D scenario, and to a lesser extent the One third each scenario, the overshoot and degrowth phases of the GWP are accentuated compared to the original Low scenario, meaning that these carbon tax scenarios lead to a worse situation. In these scenarios with a carbon tax, accelerating the technological change 
Figure 5: Simulation Outputs of Carbon Price Scenarios, 1990-2250
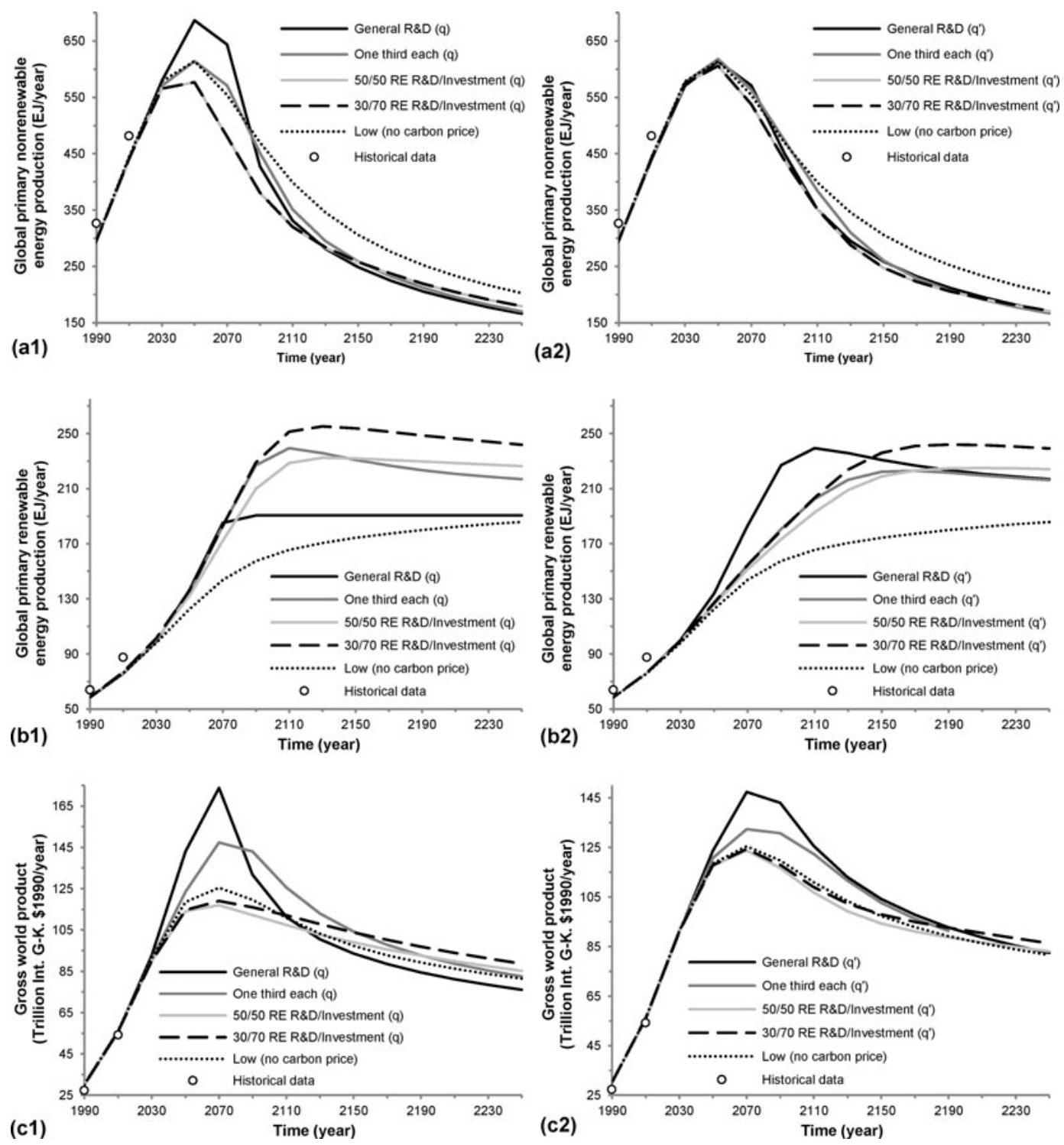

of the final sector exacerbates the nonrenewable energy lock-in of the economy. (iv) This harmful effect of technological and energy resource lock-in is lower if the less stringent carbon price $q$ ' is chosen.

These results support the criticisms made by Weyant (2011) about the price fundamentalism advanced by Nordhaus (2011). Pricing the polluting externality is not enough, and indeed additional incentives directed specifically to the renewable sector are needed to overcome its market failures, as modeled in the 50/50 and 30/70 RE R\&D/investment scenarios. Of course, further refinements of the model would be needed to correctly define the best policy option for which we do not have an optimization criterion in the current modeling state. Moreover, we have only tested scenarios in which the relative allocation shares $\beta_{i}$ of the carbon tax revenue remain constant during 
the entire simulation time, which is of course not the case in the real economy. Nevertheless, implementing the carbon tax in our model was interesting to see that it seems to represent an adequate strategy (among others surely) to attenuate, at least partially, the unfortunate future outcomes suggested by the Low scenario. ${ }^{15}$

\section{CONCLUSION}

Our model supports the idea that both the quantity of net energy supplied by energyproducing sectors to the energy-dissipative economy, and the ability of the economic system to use this energy (in fact exergy) are key elements of economic growth. To our knowledge, we are the first to develop a simple theoretical model that can be calibrated on global historical data and correctly reproduce long-term global historical trends for nonrenewable and renewable primary energy supply, aggregate technological change, and GWP. This is mainly because, unlike similar approaches, we have ensured that our theoretical model respects some of the many fundamental physical limits of the real world. These are formalized in the functional forms that we have established for the capital requirements of nonrenewable and renewable energy productions, and in the technological level of the economy formally defined as the aggregate efficiency of primary-touseful exergy conversion.

The main conclusion of this paper is clear: for an economy in which energy-producing and energy-consuming sectors are technologically consistent, and in the absence of any correction of the price system, the final efficiency of primary-to-useful exergy conversion of the global economy must be sufficiently high (above 0.35 ) in order to have a smooth future transition from nonrenewable to renewable energy that does not negatively impact economic growth. In our model, the economy cannot avoid a temporary energy lock-in (unanticipated nonrenewable energy peak occurring at a low level of renewable energy production) when this requirement for future technological level is not attained. In such circumstances the energy transition from nonrenewable to renewable energy induces an overshoot and then degrowth of the economic product. Such lock-in behavior of the economy system can be (at least partially) avoided through the implementation of a carbon price, which has also the benefit of decreasing GHG emissions from fossil-fuels use and hence mitigates climate change. Therefore, implementing a carbon price on nonrenewable energy production and recycling its revenue could help in the choice of the best development path that, at minimum, should consist in a smooth energy transition that does not negatively impact economic development. However, in its current formulation our model cannot be used to define endogenously the optimal time path of the carbon price, nor the optimal time path allocation of the carbon tax revenue among the different recycling uses. This would require to add some micro-foundations to the model in order to explain how producers and consumers receive adequate incentives to change their respective behaviors.

15. Implementing the same smoothing strategy in the Medium scenario leads to the same qualitative results. 


\section{APPENDIX}

\section{Historical Data}

Table A1: 20-years Interval Historical Estimates used for Model Calibration, 1750-2010

\begin{tabular}{ccccc}
\hline Time period (actual & $\begin{array}{c}\text { Nonrenewable } \\
\text { primary exergy } \\
\text { year) }\end{array}$ & $\begin{array}{c}\text { Renewable primary } \\
\text { exergy production } \\
\text { production (EJ/year) }\end{array}$ & $\begin{array}{c}\text { Efficiency of } \\
\text { primary-to-useful } \\
\text { exergy conversion } \\
\text { (dimensionless) }\end{array}$ & $\begin{array}{c}\text { Gross world product } \\
\text { (Billion Int. G-K. } \\
\$ 1990 / \text { year) }\end{array}$ \\
\hline (1750) & 0.00 & 19.55 & 0.0250 & 435 \\
$1(1770)$ & 0.05 & 19.65 & 0.0250 & 465 \\
$2(1790)$ & 0.20 & 19.85 & 0.0255 & 495 \\
$3(1810)$ & 0.55 & 20.50 & 0.0265 & 530 \\
$4(1830)$ & 1.00 & 21.25 & 0.0278 & 765 \\
$5(1850)$ & 2.20 & 22.05 & 0.0300 & 920 \\
$6(1870)$ & 6.00 & 22.75 & 0.0320 & 1115 \\
$7(1890)$ & 14.70 & 22.95 & 0.0360 & 1675 \\
$8(1910)$ & 31.50 & 23.20 & 0.0420 & 2550 \\
$9(1930)$ & 42.50 & 25.50 & 0.0510 & 3720 \\
$10(1950)$ & 70.30 & 28.00 & 0.0650 & 5315 \\
$11(1970)$ & 201.5 & 38.35 & 0.0800 & 13720 \\
$12(1990)$ & 326.5 & 52.30 & 0.1000 & 27350 \\
$13(2010)$ & 480.0 & 74.25 & 0.1250 & 54150 \\
\hline
\end{tabular}

Source: see text.

Figure A1: Original Data and 20-years Interval Estimates, 1750-2010: (a) Nonrenewable and Renewable Global Primary Exergy Production, (b) Efficiency of Primaryto-useful Exergy Conversion, (c) Gross World Product
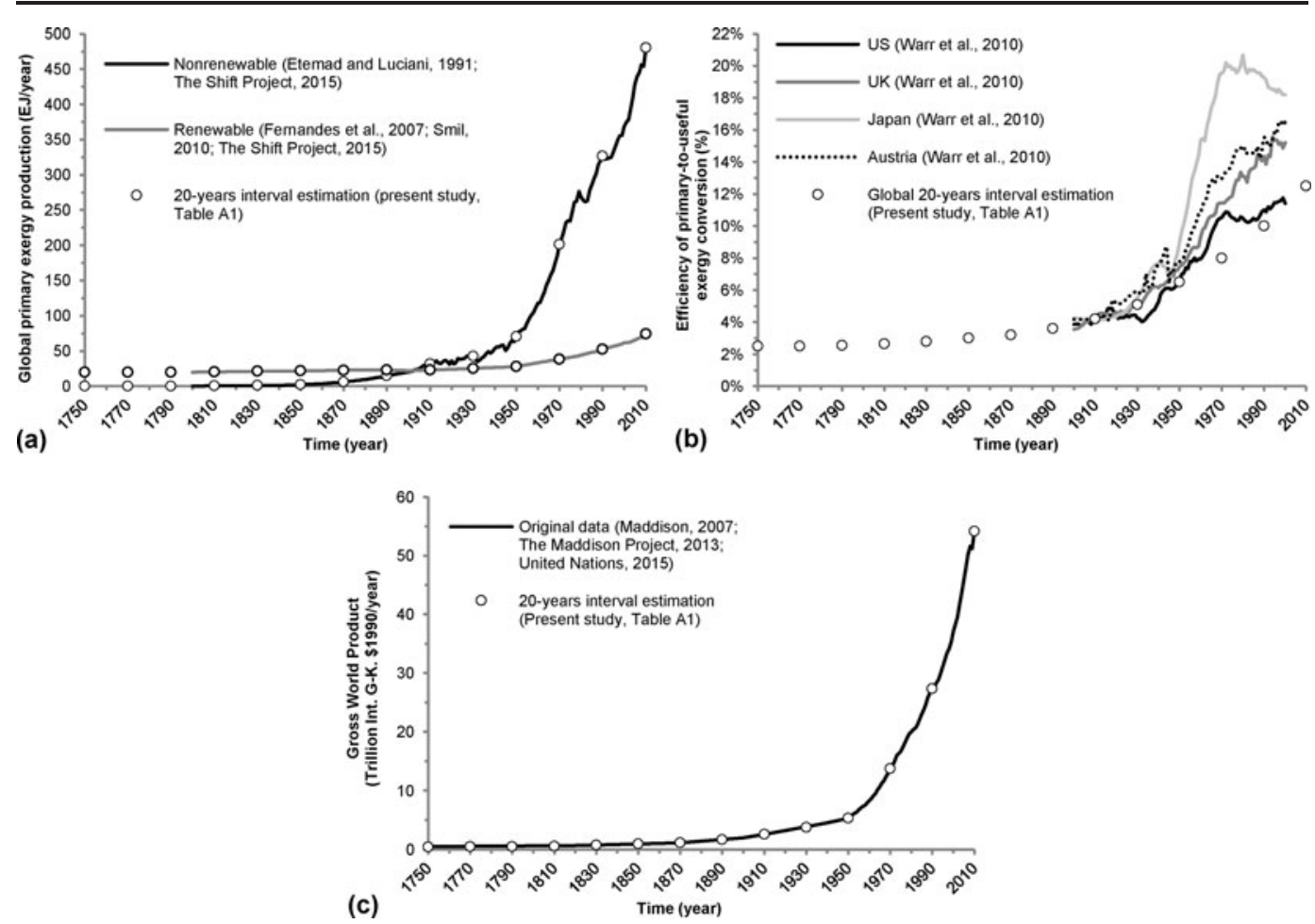

Copyright (C) 2018 by the IAEE. All rights reserved. 


\section{Parameter Values Common to All Scenarios after Calibrations}

Table A2: Set of parameter values common to all scenarios

\begin{tabular}{|c|c|c|c|}
\hline Parameter & Definition (unit) & Value & Units \\
\hline$T$ & Time horizon of the model. & 25 & dmnl \\
\hline$t_{\text {length }}$ & Time period length in real years between $t$ and $t+1$. & 20 & years \\
\hline$\lambda$ & Transformation productivity of investment goods. & 7.25 & dmnl \\
\hline$\mu$ & Annual real interest rate of the economy. & 0.03 & dmnl \\
\hline$v$ & Constant capital cost $(\mathrm{dmnl})$, with $v \equiv(1+\mu)^{t_{\text {length }} / \lambda}$ & 0.249 & dmnl \\
\hline$\alpha$ & Constant output elasticity of useful exergy. & 0.6 & dmnl \\
\hline$\sigma$ & Share of the macroeconomic investment going to $\mathrm{R} \& \mathrm{D}$. & 0.9 & dmnl \\
\hline$N$ & Number of time periods used to smooth the saving rate of the economy in $\xi_{t}$. & 4.0 & dmnl \\
\hline$\underline{A}, A_{0}$ & Initial technological level. & 0.025 & dmnl \\
\hline $\mathcal{R}$ & Ultimately recoverable resource of nonrenewable energy. & 175,500 & EJ \\
\hline$D_{0}$ & Initial unitary capital cost of NRE production. & 6.35 & $\mathrm{~B} \$ / \mathrm{EJ}$ \\
\hline$\delta$ & Rate of quality degradation of the NRE resource. & 0.225 & dmnl \\
\hline$\omega_{1}$ & Power exponent of the ratio of exploited resource $\phi_{t}$ in the cost increasing part. & 1.05 & dmnl \\
\hline$\underline{\omega_{2}}$ & Power exponent of the ratio of exploited resource $\phi_{t}$ in the cost decreasing part. & 0.05 & dmnl \\
\hline $\bar{B}$ & Initial production cost per unit of renewable energy output. & 1.35 & $\mathrm{~B} \$ / \mathrm{EJ}$ \\
\hline$\tau$ & Growth rate of $B_{t}$ towards $\underline{B}$ & 15 & dmnl \\
\hline$\eta$ & $\begin{array}{l}\text { Constant used to link the final capital cost of RE production } \underline{B} \text { to its initial value } \\
\bar{B} \text {, and to the ultimate technological level gain ratio } \bar{A} / \underline{A} \text {. }\end{array}$ & 0.25 & dmnl \\
\hline$\theta$ & Returns to scale in the NRE sector. & 0.5 & dmnl \\
\hline$\gamma$ & Returns to scale in the RE sector. & 0.5 & dmnl \\
\hline$\chi_{N R E}$ & Share of gross primary energy production self-consumed by the NRE sector. & 0.01 & dmnl \\
\hline$\chi_{R E}$ & Share of gross primary energy production self-consumed by the RE sector. & 0.01 & dmnl \\
\hline $\mathrm{H}_{0}$ & Initial (1750) capital in the final sector. & 745 & $\mathrm{~B} \$$ \\
\hline $\mathrm{Y}_{0}$ & Initial (1750) gross world product. & 435 & $\mathrm{~B} \$ / \mathrm{yr}$ \\
\hline $\mathrm{S}_{0}$ & Initial (1750) saving rate of the economy. & 0.5 & dmnl \\
\hline
\end{tabular}

\section{Nonrenewable URR}

To obtain the value of the aggregated fossil URR we use the recent work of McGlade and Ekins (2015) ant take their best estimates for oil (Gb: giga barrels), gas (Tcm: terra cubic meters), and coal (Gt: giga tonnes), which for the record are in accordance with the last IIASA Global Energy Assessment report (IIASA, 2012). For uranium (EJ: Exajoule), we aggregate the best estimate of conventional and unconventional uranium resource provided by IIASA (2012), giving the rounded value of 14,500 EJ. After conversion and aggregation, the total nonrenewable URR value retained for our simulations is $175,500 \mathrm{EJ}$ as can be seen in Table A3.

Table A3: Data used for the Aggregation of Coal, Oil, Gas and Uranium Global URR

\begin{tabular}{|c|c|c|c|}
\hline Energy resource & Global URR (diverse units) & Conversion factors (diverse units) & Global URR (EJ) \\
\hline Coal & 4085 (Gt) & & 105,000 \\
\hline $63 \%$ hard coal & $2565(G t)$ & 32.5E-9 EJ/tonne & 83,500 \\
\hline $37 \%$ lignite coal & $1520(G t)$ & 14.0E-9 EJ/tonne & 21,500 \\
\hline Oil & $5070(\mathrm{~Gb})$ & & 29,000 \\
\hline Conventional oil & $2615(G b)$ & $5.73 E-9$ EJ/barrel & 15,000 \\
\hline Unconventional oil & $2455(G b)$ & $5.73 E-9$ EJ/barrel & 14,000 \\
\hline Gas & $675(\mathrm{Tcm})$ & & 27,000 \\
\hline Conventional gas & $375(\mathrm{Tcm})$ & $40 \mathrm{EJ} / \mathrm{Tcm}$ & 15,000 \\
\hline Unconventional gas & $300(\mathrm{Tcm})$ & $40 \mathrm{EJ} / \mathrm{Tcm}$ & 12,000 \\
\hline Total fossil fuels & & & 161,000 \\
\hline Uranium & & & 14,500 \\
\hline Total nonrenewable energy & & & 175,500 \\
\hline
\end{tabular}

Source: IIASA (2012), McGlade and Ekins (2015).

Notes: URR values expressed in EJ have been rounded up to the nearest 500.

Copyright (C) 2018 by the IAEE. All rights reserved. 
Simulations Results on the Restricted 1750-2010 Period

Figure A2: Historical vs. Simulated Data, 1750-2010: (a) Primary Nonrenewable Energy Production, (b) Primary Renewable Energy Production, (c) Global Efficiency of Primary-to-useful Exergy Conversion, (d) Gross World Product
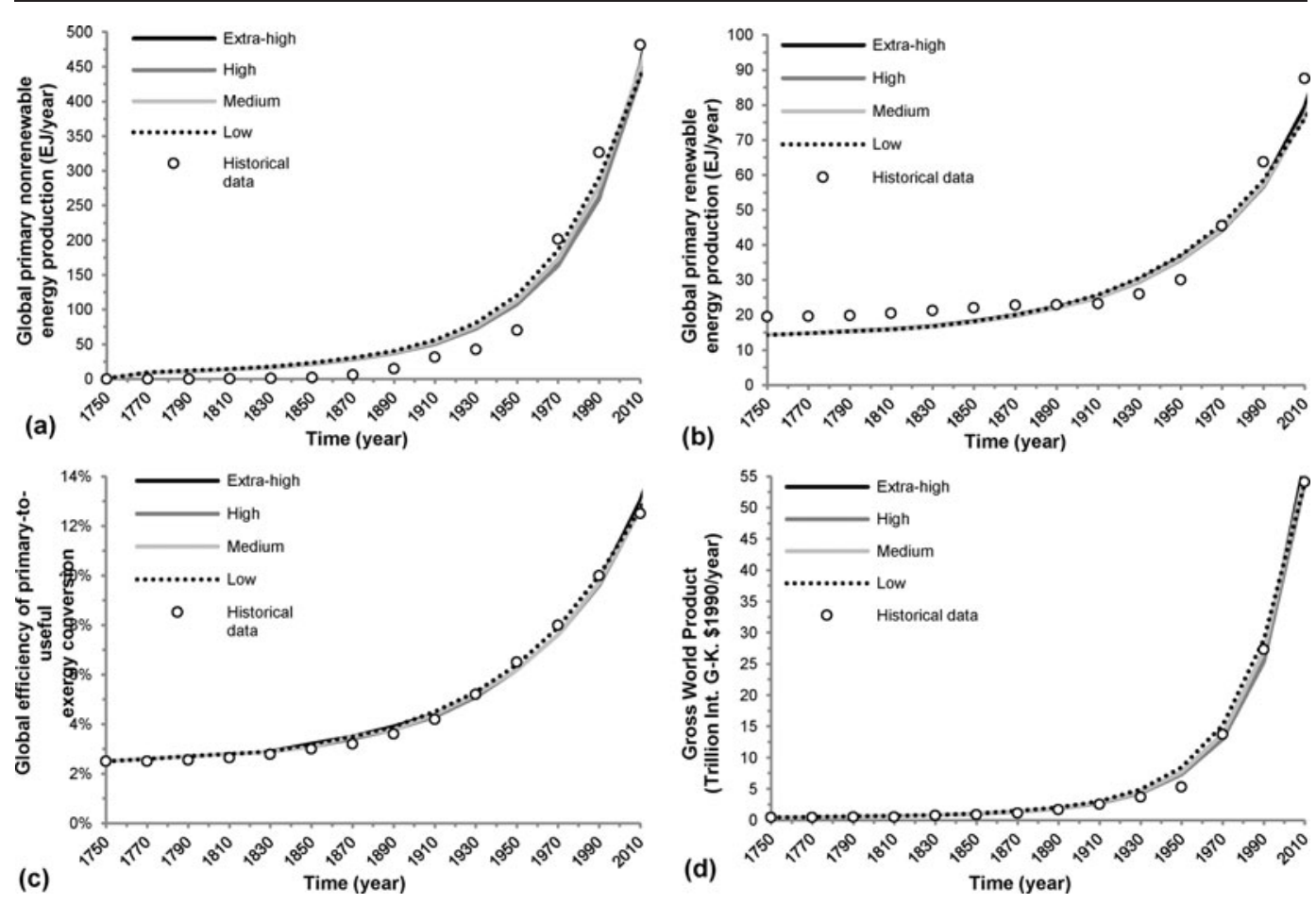

\section{ACKNOWLEDGMENTS}

The authors would like to thank Clément Bonnet, Simon Quemin, and Olivier Massol for their review of a preliminary version of this paper, particularly for their respective helpful comments regarding the carbon taxes scenarios, the simulation approach, and the definition of the technological change. We have greatly appreciated all the suggestions made by scholars at the EconomiX seminar. Three anonymous reviewers have added much to the quality of this article thanks to their insightful comments.

\section{REFERENCES}

Acemoglu, D., P. Aghion, L. Bursztyn, and D. Hemous (2012). “The environment and directed technical change.” American Economic Review 102(1): 131-166. https://doi.org/10.1257/aer.102.1.131.

Aghion, P. and P. Howitt (1992). “A model of growth through creative destruction.” Econometrica 60(2): 323-351. https:/ /doi.org/10.2307/2951599.

Ayres, R.U. (1998). "Eco-thermodynamics: economics and the second law.” Ecological Economics 26(2): 189-209. https:/ /doi.org/10.1016/S0921-8009(97)00101-8.

Ayres, R. and B. Warr (2009). The Economic Growth Engine: How Energy and Work Drive Material Prosperity. Cheltenham, UK: Edward Elgar Publishing. https://doi.org/10.4337/9781848445956. 
Boden, T.A., G. Marland, and R.J. Andres (2013). "Global, Regional, and National Fossil-Fuel $\mathrm{CO}_{2}$ Emissions.” Carbon Dioxide Information Analysis Center, Oak Ridge National Laboratory, U.S. Department of Energy, Oak Ridge, Tennessee, USA.

British Petroleum (2015). Oil reserves definition for the "Statistical Review of World Energy 2015." Available at: http:// www.bp.com/content/dam/bp/pdf/Energy-economics/statistical-review-2015/oil-reserve-definitions-bp-2015.pdf

Cleveland, C.J., R. Costanza, C.A.S. Hall., and R. Kaufman (1984). "Energy and the United States: a biophysical perspective." Science 225: 890-897. https://doi.org/10.1126/science.225.4665.890.

Dale M., S. Krumdieck, and P. Bodger (2011). "Net energy yield from production of conventional oil." Energy policy 39(11): 7095-7102. https://doi.org/10.1016/j.enpol.2011.08.021.

Dale M., S. Krumdieck, and P. Bodger (2012). "Global energy modelling - A biophysical approach (GEMBA), Part 2: Methodology." Ecological Economics 73: 158-167. https://doi.org/10.1016/j.ecolecon.2011.10.028.

Daly, H.E. (1977). Steady-State Economics. Washington, D.C.: Island Press.

Dasgupta, P.S. and G.M. Heal (1974). "The optimal depletion of exhaustible resources." Review of Economic Studies 41: 3-28. https://doi.org/10.2307/2296369.

Etemad, B. and J. Luciani (1991). World energy production, 1800-1985. Production mondiale d'énergie, 1800-1985. Genève, $\mathrm{CH}$ : Librairie Droz.

Fagnart, J.F. and M. Germain (2014). "Can the energy transition be smooth?." CEREC working paper, Université SaintLouis, Brussels, Belgium. Available at: http://centres.fusl.ac.be/CEREC/document/2014/cahier2014_11.pdf.

Fernandes, S. D., N. M. Trautmann, D. G. Streets, C. A. Roden, and T. C. Bond (2007). "Global biofuel use, 1850-2000." Global Biogeochem. Cycles 21(2): 1-15. https://doi.org/10.1029/2006GB002836.

Galor, O. (2011). Unified Growth Theory. Princeton, NJ: Princeton University Press.

Galor, O., and Weil, D. (2000). "Population, Technology, and Growth: From Malthusian Stagnation to the Demographic Transition and Beyond." American Economic Review 90(4): 806-828. https://doi.org/10.1257/aer.90.4.806.

IIASA, International Institute for Applied System Analysis (2012). Global Energy Assessment - Toward a Sustainable Future. Cambridge, UK: Cambridge University Press.

Georgescu-Roegen, N. (1971). The Entropy Law and the Economic Process. Cambridge, MA: Harvard University Press. https://doi.org/10.4159/harvard.9780674281653.

Georgescu-Roegen, N. (1979). "Energy analysis and economic valuation.” Southern Economic Journal 45(4): $1023-1058$. https://doi.org/10.2307/1056953.

Grossman, G. M. and E. Helpman (1991). "Quality ladders in the theory of growth." Review of Economic Studies 58(1): 43-61. https://doi.org/10.2307/2298044.

Hall, C.A.S. and K. A. Klitgaard (2012). Energy and the Wealth of Nations: Understanding the Biophysical Economy. New York, NY: Springer. https://doi.org/10.1007/978-1-4419-9398-4.

Hall, C.A.S., J.G. Lambert, and S. Balogh (2014). "EROI of different fuels and the implications for society." Energy Policy 64: 141-152. https://doi.org/10.1016/j.enpol.2013.05.049.

Hartley, P., K.B. Medlock, T. Temzelides, and X. Zhang (2016). "Energy sector innovation and growth: an optimal energy crisis." The Energy Journal 37(1): 233-258. https://doi.org/10.5547/01956574.37.1.phar.

Honnery, D. and P. Moriarty (2009). "Estimating global hydrogen production from wind." International Journal of Hydrogen Energy 34(2): 727-736. https://doi.org/10.1016/j.ijhydene.2008.11.001.

Hoogwijk, M., B. de Vries, and W. Turkenburg (2004). "Assessment of the global and regional geographical, technical and economic potential of onshore wind energy." Energy Economics 26(5): 889-919. https://doi.org/10.1016/j.eneco.2004. 04.016.

Jouvet, P.-A., and I. Schumacher (2011). "Learning-by-doing and the costs of a backstop for energy transition and sustainability." Ecological Economics 73: 122-132. https://doi.org/10.1016/j.ecolecon.2011.10.007.

King, C.W., J.P. Maxwell, A. Donovan (2015). “Comparing world economic and net energy metrics, Part 1: single technology and commodity perspective.” Energies 8: 12949-12974. https://doi.org/10.3390/en81112346.

Kümmel, R. (1989). "Energy as a Factor of Production and Entropy as a Pollution Indicator in Macroeconomic Modeling." Ecological economics 1:161-180. https://doi.org/10.1016/0921-8009(89)90003-7.

Kümmel, R. (2011). The Second Law of Economics: Energy, Entropy, and the Origins of Wealth. New York, NY: Springer. https://doi.org/10.1007/978-1-4419-9365-6.

Lucas, R.E. (1988). "On the mechanics of economic development.” Journal of Monetary Economics 22: 3-42. https:// doi.org/10.1016/0304-3932(88)90168-7.

Maddison, A. (2007). Contours of the World Economy, 1-2030 AD. Oxford, UK: Oxford University press.

McGlade, C. and P. Ekins (2015). "The geographical distribution of fossil fuels unused when limiting global warming to $2^{\circ}$ C." Nature 517: 187-190. https://doi.org/10.1038/nature14016.

Copyright (C) 2018 by the IAEE. All rights reserved. 
Meadows, D.H., D.L. Meadows, J. Randers, and W.W. Behrens (1972). The Limits to Growth: A Report for the Club of Rome's Project on the Predicament of Mankind. New York, NY: Universe Books.

Murphy, D.J. and C.A.S Hall (2011). "Energy return on investment, peak oil, and the end of economic growth.” In Ecological Economics Reviews. R. Costanza, K. Limburg \& I. Kubiszewski, Eds. Ann. N.Y.Acad. Sci. 1219: 52-72. https://doi.org/ 10.1111/j.1749-6632.2010.05940.x.

Nordhaus, W. (2011). "Designing a friendly space for technological change to slow global warming." Energy Economics 33(4): 665-673. https://doi.org/10.1016/j.eneco.2010.08.005.

NREL, (2010a). Western Wind Dataset. National Renewable Energy Laboratory.

NREL, (2010b). National Solar Radiation Database. National Renewable Energy Laboratory.

Odum, H.T. (1971). Environment, Power, and Society. New York, NY: Wiley-Interscience.

Odum, H.T. (1973). "Energy, ecology and economics." AMBIO 2(6): 220-227.

Rebelo, S. (1991). "Long-run policy analysis and long-run growth.” Journal of Political Economy 99(3): 500-521. https:// doi.org/10.1086/261764.

Romer, P. M. (1986). "Increasing returns and long run growth.” Journal of Political Economy 94(5): 1002-1037. https:// doi.org/10.1086/261420.

Romer, P.M. (1987). "Growth based on increasing returns due to specialization.” American Economic Review 77(2): 5662.

Romer, P. M. (1990). “Endogenous technical change.” Journal of Political Economy 98(5): 71-102. https://doi.org/10.1086/ 261725.

Smil, V. (2010). Energy Transitions: History, Requirements, Prospects. Santa Barbara, CA: Praeger Publishers Inc.

Smulders, S. (1995). "Entropy, Environment, and Endogenous Economic Growth." International Tax and Public Finance 2: 319-340. https://doi.org/10.1007/BF00877504.

Solow, R.M. (1956). “A Contribution to the Theory of Economic Growth.” Quarterly Journal of Economics 70(1): 65-94. https://doi.org/10.2307/1884513.

Solow, R. M. (1974). "The Economics of Resources or the Resources of Economics." American Economic Review 64(2): 1-14. https://doi.org/10.1057/9780230523210_13.

Sorrell, S., J. Spiers, R. Bentley, A. Brandt, and R. Miller (2010). "Global oil depletion: a review of the evidence.” Energy Policy 38(9): 5290-5295. https://doi.org/10.1016/j.enpol.2010.04.046.

Stiglitz, J.E. (1974). "Growth with exhaustible natural resources, efficient and optimal growth paths." Review of Economic Studies 41: 123-137. https://doi.org/10.2307/2296377.

Swan, T. (1956). "Economic growth and capital accumulation." Economic Record 32(2): 334-361. https://doi.org/10.1111/ j.1475-4932.1956.tb00434.x.

Tahvonen O. and S. Salo (2001). "Economic growth and transitions between renewable and nonrenewable energy resources." European Economic Review 45(8): 1379-1398. https://doi.org/10.1016/S0014-2921(00)00062-3.

The Maddison Project (2013). GDP per Capita 1-2010 AD. Available at: http://www.ggdc.net/maddison/maddison-project/ home.htm.

The Shift Project (2015). Historical Energy Production Statistics. Available at: http://www.tsp-data-portal.org/Energy-Production-Statistics\#tspQvChart.

Tsur Y., and A. Zemel (2005). "Scarcity, growth and R\&D." Journal of Environmental Economics and Management 49(3): 484-499. https://doi.org/10.1016/j.jeem.2004.07.001.

United Nations (2015). Department of Economic and Social Affairs, Population Division, World Population Prospects, the 2015 Revision. Available at: http://esa.un.org/unpd/wpp/Download/Standard/Population/.

EIA, US Energy Information Administration (2014). International Energy Statistics. Available at: http://www.eia.gov/cfapps/ ipdbproject/IEDIndex3.cfm.

Warr, B., R.U. Ayres, N. Eisenmenger, F. Krausmann, and H. Schandl (2010)."Energy use and economic development: A comparative analysis of useful work supply in Austria, Japan, the United Kingdom and the US during 100 years of economic growth.” Ecological Economics 69: 1904-1917. https://doi.org/10.1016/j.ecolecon.2010.03.021.

Warr, B. and R.U. Ayres (2012). "Useful work and information as drivers of economic growth." Ecological Economics 73: 93-102. https://doi.org/10.1016/j.ecolecon.2011.09.006.

Weyant, J.P. (2011). "Accelerating the development and diffusion of new energy technologies: Beyond the 'valley of death'." Energy Economics 33(4): 674-682. https://doi.org/10.1016/j.eneco.2010.08.008.

World Bank (2016). World Bank Indicators, Gross savings (\% of GDP). Available at: http://data.worldbank.org/indicator/ NY.GNS.ICTR.ZS. 
